\title{
Scoping assessment of building vibration induced by railway traffic
}

\author{
D. López-Mendoza ${ }^{\mathrm{a}}$, A. Romero ${ }^{\mathrm{a}}$, D.P. Connolly ${ }^{\mathrm{b}}$, P. Galvín ${ }^{\mathrm{a}, *}$ \\ ${ }^{a}$ Escuela Técnica Superior de Ingeniería, Universidad de Sevilla, Camino de los Descubrimientos, 41092 Sevilla, Spain \\ ${ }^{b}$ Institute for Infrastructure and Environment, Heriot Watt University, Edinburgh, UK
}

\begin{abstract}
This work presents a scoping model to predict ground-borne railway vibration levels within buildings considering soil-structure interaction (SSI). It can predict the response of arbitrarily complex buildings in a fraction of the time typically required to analyse a complex SSI problem, and thus provides a practical tool to rapidly analyse the vibration response of numerous structures near railway lines. The tool is designed for use in cases where the ground-borne vibration is known, and thus can be used as model input. Therefore in practice, for the case of a new line, the ground motion can be computed numerically, or alternatively, for the case of new buildings to be constructed near an existing line, it can be recorded directly (e.g. using accelerometers) and used as model input. To achieve these large reductions in computational time, the model discretises the ground-borne vibration in the free field into a frequency range corresponding to the modes that characterize the dynamic building response. After the ground-borne response spectra that corresponds with the incident wave field is estimated, structural vibration levels are computed using modal superposition, thus avoiding intensive soil-structure interaction computations. The model is validated using a SSI problem and by comparing results against a more complex finite element-boundary element model. Finally, the new scoping model is then used to analyse the effect of soil properties, building height, train speed and distance between the building and the track on structural-borne vibration. The results show that the scoping model provides a powerful tool for use during the early design stages of a railway system when a large number of structures require analysis.

Keywords: Scoping assessment, Modal superposition, Railway traffic, High speed rail, Building vibrations, Ground-borne vibrations, Structural vibration, Railroad vibration, Environmental Impact Assessment (EIA)
\end{abstract}

\section{Introduction}

The expansion of high speed rail (HSR) has been decisive for economic development across the world, however this growth has also led to an increase in those effected by ground-borne vibrations from railways [1].

\footnotetext{
* Corresponding author

Email address: pedrogalvin@us.es (P. Galvín)
} 
The negative effects of this vibration are numerous and it is thus addressed in international standards. One of these standards is ISO2631 [2, 3], where indoor, whole-body human exposure to vibration is evaluated in the frequency range, $1 \mathrm{~Hz}$ to $80 \mathrm{~Hz}$. The vibration evaluation is based on the root-mean-square (RMS) value of the acceleration in the three orthogonal directions. Additionally, ISO14837 [4], a dedicated standard for the railway sector, is currently under development. This presents an overview of ground-borne vibration due to railway traffic, prediction techniques, experimental measurement, evaluation criteria and also mitigation.

It also discusses numerical modelling, including two-and-a-half-dimensional (2.5D) and three-dimensional (3D) models, which are referred to as detailed design models and can be used during the construction stage of new lines. 2.5D models are based on the assumption that the problem is homogeneous in the track direction, thus reducing the degrees of freedom. Several authors [5-13] have presented 2.5D methodologies to predict vibrations produced by railway traffic using boundary element (BEM)- finite element (FEM) coupled formulations. Three-dimensional models account for local soil discontinuities, underground constructions and structures that break the uniformity of the geometry along the track line [14-18], however, are more computationally expensive.

At the earlier stages of development for a new railway line, simpler and quicker methodologies are desirable. These models, called scoping models [4], allow engineers to asses long lengths of track in a reduced computational time, because typically, the train-track-soil interaction (source and propagation problem) is decoupled from soil-structure interaction (immission problem). Coulier et. al [19] studied the effect of assuming an uncoupled approach in a ballasted track and they concluded that it can be neglected for distances to the track longer than six times the Rayleigh wave length, thus validating this assumption.

Nelson and Sauernmann [20] presented a simple in-situ testing methodology based on impact-testing procedures to characterize soil vibrations and vehicle-track systems. Alternatively, Madshus et al. [21] developed a semi-empirical model from the statistical analysis of railway vibration measurements in Norway and Sweden. This model was used to study low frequency vibrations due to high speed trains (HST) on soft soils. Rossi and Nicolini [22] also presented an approach to predict train-induced vibration considering different train types, train speeds, track properties and distances to the track. The analytical expressions of the model were calibrated by experimental data. With et al. [23] proposed a scoping model to compute running RMS values of velocity based on the wheel force, the train speed and the distance to the track, while the Federal Railroad Administration (FRA) and the Federal Transit Administration (FTA) of the U.S. Department of Transportation have proposed empirical procedures to predict vibration levels due to railway traffic [24, 25]. Verbraken et al. [26] verified by means of a numerical method the assumptions introduced in these approaches. Later, Kuo et al. [27] developed two models using a combination of field measurements and numerical methods based on the use of separate source and propagation mechanism, and implemented them using the definitions proposed in References [24, 25]. Auersch [28] studied building induced vibrations using a simple soil-wall-floor model based on an empirical transfer fuction obtained from the characteristics 
of the structure. A soil modelled using a spring and a viscous damper was used to evaluate the effects of soil-structure interaction. François et al. [29] developed an analysis of building induced vibrations by employing simplified methods that discard SSI, but take into account the relative stiffness between the building and the soil. Recently, Conolly et al. [30, 31] presented a scoping tool, called Scoperail, to predict in-door noise in buildings and structural vibrations values due to high speed trains. A 3D FEM model was used to generate vibration records for a wide range of train speeds and soil types, and these results were combined with empirical factors in order to compute vibrations due to train passages.

The present paper builds upon these previous approaches and proposes a scoping methodology to evaluate building induced vibrations at the early development stage of railway lines using modal superposition and considering SSI. Free-field response due to train passages is the required model input data, and can be obtained from numerical models and experimental records, including conventional, freight and high speed trains. Therefore the model can be used to predict structural vibrations in the cases of both new and existing lines. The proposed method allows to assess the building response with a very low computational effort, and can be used in a general purpose FEM program. This paper is organized as follows. First, the scoping model is presented. Next, the proposed model is numerically validated comparing with a more comprehensive methodology. Finally, the effect of the soil properties, the building height, the train speed and the distance from the track to the building on the results from the scoping model is analysed.

\section{Numerical model}

This section describes the proposed scoping model. The dynamic analysis is carried out by modal superposition [32] of the structure subjected to support excitation, with the aim of computing the overall RMS value of the response due to an incident wavefield.

The dynamic equilibrium equation of a structure can be written as:

$$
\mathbf{M} \ddot{\mathbf{u}}_{t}(t)+\mathbf{C} \dot{\mathbf{u}}_{t}(t)+\mathbf{K} \mathbf{u}_{t}(t)=\mathbf{F}
$$

where $\mathbf{M}, \mathbf{C}$ and $\mathbf{K}$ are the mass, damping and stiffness matrices, respectively. $\mathbf{u}_{t}$, $\dot{\mathbf{u}}_{t}$, and $\ddot{\mathbf{u}}_{t}$ are the total displacements, velocities and accelerations, respectively, and $\mathbf{F}$ represents the external force. The total displacement can be decomposed as the sum of the ground motion $\mathbf{u}_{g}$ and that due to the structure deformation $\mathbf{u}$ :

$$
\mathbf{u}_{t}(t)=\mathbf{u}(t)+\mathbf{r} \mathbf{u}_{g}(t)
$$

where the influence matrix $\mathbf{r}$ defines the wave incidence on the structure.

Substituting the Equation (2) into the Equation (1), and considering that the ground motion $\mathbf{u}_{g}$ does not produce either viscous force $\left(\mathbf{C} \dot{\mathbf{u}}_{g}=\mathbf{0}\right)$ or elastic force $\left(\mathbf{K r} \mathbf{u}_{g}=\mathbf{0}\right)$, the following equation can be obtained:

$$
\mathbf{M} \ddot{\mathbf{u}}(t)+\mathbf{C} \dot{\mathbf{u}}(t)+\mathbf{K u}(t)=-\mathbf{M r} \ddot{\mathbf{u}}_{g}(t)
$$


The displacement vector $\mathbf{u}$ is obtained by modal superposition as:

$$
\mathbf{u}(t)=\sum_{i=1}^{N} \sum_{j=1}^{3} \phi_{i} q_{i}^{j}
$$

where $\phi_{i}$ is the $i$-th mode shape, $q_{i}^{j}$ the $i$-th modal amplitude due to a ground motion at direction $j$ and $N$ is the number of modes considered to describe the structural response.

Then, Equation (3) can be rewritten for each direction $j$ by the substitution of Equation (4) and premultipliying by the mode shape transpose vector $\phi_{j}^{T}$ :

$$
\sum_{i=1}^{N}\left[\boldsymbol{\phi}_{j}^{T} \mathbf{M} \phi_{i} \ddot{q}_{i}^{j}(t)+\boldsymbol{\phi}_{j}^{T} \mathbf{C} \phi_{i} \dot{q}_{i}^{j}(t)+\boldsymbol{\phi}_{j}^{T} \mathbf{K} \boldsymbol{\phi}_{i} q_{i}^{j}(t)\right]=-\boldsymbol{\phi}_{j}^{T} \mathbf{M r} \ddot{\mathbf{u}}_{g}(t)
$$

Equation (5) can be decomposed into a system of $N$ uncoupled equations taking into account the mode shape orthogonality condition with respect to the stiffness and mass matrices. Also, it can be assumed that this condition can be applied to the damping matrix. Equation (5) then becomes:

$$
\ddot{q}_{i}^{j}(t)+4 \pi \zeta_{i} f_{i} \dot{q}_{i}^{j}(t)+4 \pi^{2} f_{i}^{2} q_{i}^{j}(t)=-\Gamma_{i}^{j} \ddot{u}_{g}^{j}(t)
$$

with

$$
\Gamma_{i}^{j}=\frac{\phi_{i}^{T} \mathbf{M} r^{j}}{\phi_{i}^{T} \mathbf{M} \phi_{i}}
$$

where $f_{i}$ is the natural frequency, $\zeta_{i}$ is the damping ratio, and $\Gamma_{i}^{j}$ is the modal participation factor for the $i$-th mode at direction $j$.

The modal amplitude $q_{i}^{j}$ can be written as:

$$
q_{i}^{j}(t)=\Gamma_{i}^{j} \xi_{i}^{j}(t)
$$

Introducing Equation (8) in Equation (6) yields:

$$
\ddot{\xi}_{i}^{j}(t)+4 \pi \zeta_{i} f_{i} \dot{\xi}_{i}^{j}(t)+4 \pi^{2} f_{i}^{2} \xi_{i}^{j}(t)=-\ddot{u}_{g}^{j}(t)
$$

The solution of Equation (9) can be computed by means of the Duhamel's integral as [32]:

$$
\xi_{i}^{j}(t)=\frac{1}{f_{d i}} \int_{0}^{t}-\ddot{u}_{g}^{j} e^{-2 \pi \zeta_{i} f_{i}(t-\tau)} \sin \left(f_{d i}(t-\tau)\right) d \tau
$$

where $f_{d i}=f_{i} \sqrt{1-\zeta_{i}^{2}}$ is the damped natural frequency. Equation (10) is solved using the generalized single solved (GSSSS) integration algorithm U0-V0 developed by Zhou and Tamma [33]. This algorithm accurately calculates the low-frequency roots of Equation (10).

Once the modal amplitude is obtained, the structural response can be computed from Equations (2) and (4). Different international standards evaluate structural vibration level, such as standard ISO 2631 [2] which defines the overall RMS value of the frequency-weighted acceleration, or alternatively, the velocity 
decibel (VdB) metric based on the running RMS value of the velocity [34]. Since the frequency weighting depends on the corresponding standard, it is not considered in the present work. Next, the procedure to asses the overall RMS value of the acceleration is developed. The VdB metric can also be estimated using a similar methodology.

The overall RMS value of the acceleration response is calculated as:

$$
a_{R M S}=\sqrt{\frac{1}{T} \int_{0}^{T} \ddot{\mathbf{u}}_{t}^{2}(t) d t}
$$

where $T$ is the characteristic period defined by the DIN 45672-2 standard [35] where the structural response is assumed to be stationary. Then, the RMS value is obtained, accounting for the previously computed $\mathbf{u}_{t}(t)$ from Equations (2) and (4):

$$
a_{R M S}=\sqrt{\frac{1}{T} \sum_{n=1}^{M}\left(\sum_{j=1}^{3}\left(r^{j} \ddot{u}_{g}^{j}\left(t_{n}\right)+\sum_{i=1}^{N} \phi_{i} \Gamma_{i}^{j} \ddot{\xi}_{i}^{j}\left(t_{n}\right)\right)\right)^{2} \Delta t}
$$

being $t=t_{1}, t_{2}, \ldots, t_{n}, \ldots, t_{M}$ with $\Delta t=t_{n}-t_{n-1}$.

After expanding, Equation (12) can be written as:

$a_{R M S}=\sqrt{\frac{1}{T} \sum_{n=1}^{M}\left(\sum_{j=1}^{3}\left(r^{j} \ddot{u}_{g}^{j}\left(t_{n}\right)\right)^{2}+\left(\sum_{i=1}^{N} \sum_{j=1}^{3}\left(\phi_{i} \Gamma_{i}^{j} \ddot{\xi}_{i}^{j}\left(t_{n}\right)\right)\right)^{2}+\sum_{i=1}^{N} \sum_{j=1}^{3} \sum_{k=1}^{3}\left(2 r^{k} \boldsymbol{\phi}_{i} \Gamma_{i}^{j} \ddot{u}_{g}^{k}\left(t_{n}\right) \ddot{\xi}_{i}^{j}\left(t_{n}\right)\right)\right) \Delta t}$

Bearing in mind $\frac{T}{\Delta t}=M$, Equation (13) is expressed in a compact form as:

$$
a_{R M S}=\sqrt{H_{g}+H_{b}+H_{g b}}
$$

with

$$
\begin{gathered}
H_{g}=\frac{1}{M} \sum_{n=1}^{M} \sum_{j=1}^{3}\left(r^{j} \ddot{u}_{g}^{j}\left(t_{n}\right)\right)^{2} \\
H_{b}=\frac{1}{M} \sum_{n=1}^{M}\left(\sum_{i=1}^{N} \sum_{j=1}^{3}\left(\phi_{i} \Gamma_{i}^{j} \ddot{\xi}_{i}^{j}\left(t_{n}\right)\right)\right)^{2} \\
H_{g b}=\frac{1}{M} \sum_{n=1}^{M} \sum_{i=1}^{N} \sum_{j=1}^{3} \sum_{k=1}^{3}\left(2 r^{k} \phi_{i} \Gamma_{i}^{j} \ddot{u}_{g}^{k}\left(t_{n}\right) \ddot{\xi}_{i}^{j}\left(t_{n}\right)\right)
\end{gathered}
$$

$H_{g}, H_{b}$ and $H_{g b}$ represent the contributions to the RMS value of the ground motion, the structural response and the coupling between both terms, respectively.

The generalization of Parseval's theorem for two time functions $f(t)$ and $g(t)$ whose Fourier transforms are $F(\omega)$ and $G(\omega)$ entails [36]:

$$
\int_{-\infty}^{+\infty} f(t) g^{*}(t) d t=\frac{1}{2 \pi} \int_{-\infty}^{+\infty} F(\omega) G^{*}(\omega) d \omega
$$


where * means complex conjugate and $\omega$ is the angular frequency.

The application of the theorem for equally-spaced samples of two real functions $f\left(t_{n}\right)$ and $g\left(t_{n}\right)$ can be written as:

$$
\sum_{n=1}^{M} f\left(t_{n}\right) g\left(t_{n}\right)=\frac{1}{M} \sum_{n=1}^{M}\left[\operatorname{sgn}\left\{\Re\left(F\left(f_{n}\right) G^{*}\left(f_{n}\right)\right)\right\}\left|F\left(f_{n}\right) G^{*}\left(f_{n}\right)\right|\right]
$$

where $f_{n}=\frac{n}{M \Delta t}$.

The terms $H_{g}, H_{b}$ and $H_{g b}$ (Equations (15-17)) can be computed from Equation (19) as:

$$
\begin{gathered}
H_{g}=\frac{1}{M^{2}} \sum_{n=1}^{M} \sum_{j=1}^{3} r^{j^{2}}\left|\ddot{U}_{g}^{j}\left(f_{n}\right)\right|^{2} \\
H_{b}=\frac{1}{M^{2}} \sum_{n=1}^{M}\left|\sum_{i=1}^{N} \sum_{j=1}^{3}\left(\phi_{i} \Gamma_{i}^{j} \ddot{\Xi}_{i}^{j}\left(f_{n}\right)\right)\right|^{2} \\
H_{g b}=\frac{1}{M^{2}} \sum_{n=1}^{M} \sum_{i=1}^{N} \sum_{j=1}^{3} \sum_{k=1}^{3}\left[2 r^{k} \phi_{i} \Gamma_{i}^{j} \operatorname{sgn}\left\{\Re\left(\ddot{U}_{g}^{k}\left(f_{n}\right) \ddot{\Xi}_{i}^{j *}\left(f_{n}\right)\right)\right\}\left|\ddot{U}_{g}^{k}\left(f_{n}\right) \ddot{\Xi}_{i}^{j *}\left(f_{n}\right)\right|\right]
\end{gathered}
$$

where $\ddot{U}_{g}^{j}\left(f_{n}\right)$ and $\ddot{\Xi}_{i}^{j}\left(f_{n}\right)$ are the Discrete Fourier transforms of $\ddot{u}_{g}^{j}\left(t_{n}\right)$ and $\ddot{\xi}_{i}^{j}\left(t_{n}\right)$.

The present model uses some assumptions in the terms $H_{b}$ and $H_{g b}$ of the Equation (14) in order to developed a simple procedure that can be easily used in a general purpose FEM commercial program. The first simplification is that the cross product term $H_{g b}$ is neglected. It is based on the assumption that the structural response $\ddot{\Xi}_{i}^{j}\left(f_{n}\right)$ amplifies the soil motion $\ddot{U}_{g}^{k}\left(f_{n}\right)$ and, therefore, the term $\ddot{U}_{g}^{k}\left(f_{n}\right) \ddot{\Xi}_{i}^{j *}\left(f_{n}\right)$ is much lower than $\ddot{\Xi}_{i}^{j}\left(f_{n}\right)$.

Moreover, the Equation (21) can be expanded as follows:

$$
H_{b}=\frac{1}{M^{2}} \sum_{n=1}^{M}\left[\sum_{i=1}^{N} \sum_{j=1}^{3}\left(\phi_{i} \Gamma_{i}^{j}\left|\ddot{\Xi}_{i}^{j}\left(f_{n}\right)\right|\right)^{2}+\sum_{i=1}^{N} \prod_{\substack{j=1 \\ k=1 \\ j \neq k}}^{3}{\phi_{i}}^{2} \Gamma_{i}^{j} \ddot{\Xi}_{i}^{j}\left(f_{n}\right) \Gamma_{i}^{k} \ddot{\Xi}_{i}^{k *}\left(f_{n}\right)+\sum_{j=1}^{3} \sum_{k=1}^{3} \prod_{\substack{i=1 \\ l=1 \\ i \neq l}}^{N} \phi_{i} \Gamma_{i}^{j} \ddot{\Xi}_{i}^{j}\left(f_{n}\right) \phi_{l} \Gamma_{l}^{k} \ddot{\Xi}_{l}^{k *}\left(f_{n}\right)\right]
$$

In the proposed methodology only the first term of Equation (23) is considered. This assumption is based on: i) the cross product $\Gamma_{i}^{j} \Gamma_{i}^{k}$ between the modal participation factor for the $i$-th mode at different directions $j$ and $k$ can be disregarded, and ii) since the functions $\ddot{\Xi}_{i}^{j}\left(f_{n}\right)$ and $\ddot{\Xi}_{l}^{k *}\left(f_{n}\right)$ are frequency responses of onedegree-of freedom systems, the cross product $\Xi_{i}^{j}\left(f_{n}\right) \Xi_{l}^{k *}\left(f_{n}\right)$ can be neglected if the modes are well separated and lightly damped. In the next section, the study of the uncertainties due to the simplifications carried out in the terms $H_{b}$ and $H_{g b}$ will be studied.

Then, the overall RMS value of the acceleration (Equation (14)) is given by:

$$
a_{R M S}=\sqrt{H_{g}+H_{b}^{\prime}}
$$


being $H_{b}^{\prime}=\sum_{i=1}^{N} H_{b i}^{\prime}$, with $H_{b i}^{\prime}=\phi_{i}^{2} \sum_{j=1}^{3}\left(\Gamma_{i}^{j} \Lambda_{i}^{j}\right)^{2}$. In the previous expression:

$$
\Lambda_{i}^{j}=\frac{1}{M} \sqrt{\sum_{n=1}^{M}\left|\ddot{\Xi}_{i}^{j}\left(f_{n}\right)\right|^{2}}
$$

represents the ground-borne response spectra.

The spectra defined in Equation 25 allows for straightforward integration within commercial FEM software, by solving a response spectrum analysis (RSA) [32], where the input is the ground-borne response spectra $\Lambda_{i}^{j}\left(f_{i}\right)$. The result of the RSA can be used to obtain the contribution to the response of the structural deformation $H_{b}^{\prime}$. The contribution of the ground motion should be added according to Equation (24).

The contribution of the $i$-th mode to the overall RMS value of the acceleration can be estimated from Equation (24) as:

$$
C_{i}=\sqrt{H_{b i}^{\prime}}
$$

In order to represent the structure's dynamic behaviour with accuracy, the proposed model calculates and combines the response for only those modes at frequencies $\left(f_{k}\right)$ which meet the criterion:

$$
\max \left(\frac{\Gamma_{k}^{j^{2}}}{\sum_{i=1}^{N} \Gamma_{i}^{j^{2}}}\right) \geq \varepsilon \quad j=1,2,3
$$

where $\varepsilon$ is the required tolerance.

SSI is integrated into the proposed scoping model by adding spring $k_{f}$ and damper $c_{f}$ elements to the foundation of the building model. Alternative simplified solutions, depending on the type of foundation can be found in previous literature [28, 37-39]: isolated footing, continuous footing, isolated pile and pile group. In this work it was considered the following correlation for shallow foundations from the model presented by Auersch [28]: $k_{f}=3.4 G_{s} \sqrt{A_{f}}$ and $c_{f}=1.6 \sqrt{G_{s} \rho_{s} A_{f}}$, where $G_{s}$ and $\rho_{s}$ are the shear modulus and the mass density of the soil, respectively, and $A_{f}$ is the foundation area.

\section{Numerical verification}

\subsection{Scoping model validation}

The proposed model was numerically validated by analysing the dynamic behaviour of a building due to an incident wavefield. To do so, the structural response as computed by the proposed scoping model was compared with that obtained by the SSIFiBo toolbox [40] based on a 3D time domain BEM-FEM methodology.

The structure was a three-storey building with dimensions $14.4 \mathrm{~m} \times 10.8 \mathrm{~m} \times 9 \mathrm{~m}$ (Figure 1) [14]. It consisted of eight columns of width $0.3 \mathrm{~m} \times 0.3 \mathrm{~m}$, and a core wall with thickness of $0.15 \mathrm{~m}$. The floors were 
modelled as slabs with a thickness of $0.2 \mathrm{~m}$. The foundation was considered as a $h_{f}=0.3 \mathrm{~m}$ thick slab. All the structural elements consisted of concrete with a Young's modulus $E_{c}=30 \times 10^{9} \mathrm{~N} / \mathrm{m}^{2}$, Poisson's ratio $\nu_{c}=0.2$ and density $\rho_{c}=2500 \mathrm{~kg} / \mathrm{m}^{3}$. Structural damping of $\zeta=0.02$ was used for all modes that contributed to the building response. In order to discretise the structure 180 two-node Euler-Bernoulli and 2118 four-node shell elements were used. The element size was small enough to adequately represent the structure dynamic behaviour below a maximum frequency of $f_{\max }=80 \mathrm{~Hz}$. The minimum wave length of the bending floor waves was given by $\lambda=\sqrt{2 \pi}\left(\frac{D}{\rho_{c} h_{f}}\right)^{(1 / 4)} / f_{\max }=3.6 \mathrm{~m}$, where $D=E_{c} h_{f}^{3}\left(1-\nu_{c}^{2}\right) / 12$ was the bending stiffness of the floors. An element size of $l=0.6 \mathrm{~m}$ was used, resulting in 6 elements per wavelength.

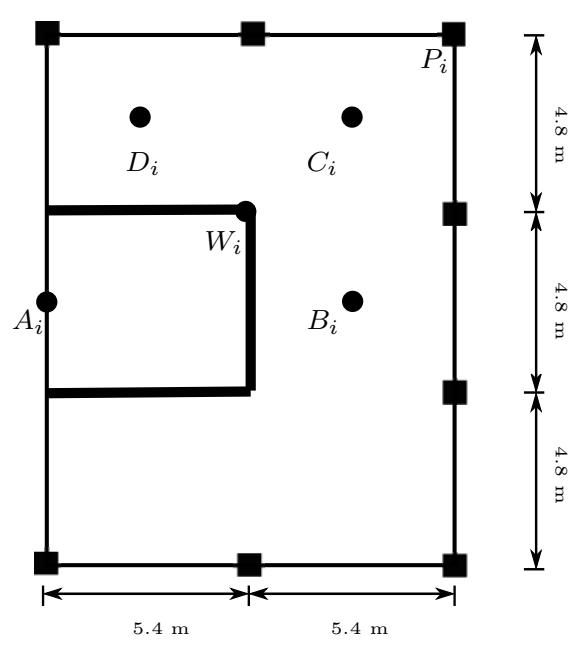

Figure 1: Building plant geometry.

The building was founded on a homogeneous soil with the following properties: $\mathrm{P}$-wave velocity $c_{p}=$ $300 \mathrm{~m} / \mathrm{s}$, S-wave velocity $c_{s}=150 \mathrm{~m} / \mathrm{s}$, material damping $\zeta_{s}=0.06$ and density $\rho_{s}=1750 \mathrm{~kg} / \mathrm{m}^{3}$. Computations were solved using a time step $\Delta t=0.002 \mathrm{~s}$ according to the stability criterion for the time domain formulation of the SSIFiBo toolbox [40]. The incident wave field corresponded with an uniform vertical displacement $\mathbf{u}_{0}=\delta(t) \mathrm{m}$, where $\delta$ was the Dirac delta function.

In the case of the scoping model, the dynamic behaviour of the building was computed using the superposition of the dominant modes. A tolerance of $\varepsilon=0.001$ was considered. Figure 2 shows the bending mode shapes of the floors for the building on foundation springs at a frequency range between $0 \mathrm{~Hz}$ and $125 \mathrm{~Hz}$.

Figure 3 shows the one-third octave band spectra content of the vertical relative accelerations $\ddot{\mathbf{u}}(t)$ at the observation points located in every floor obtained using the SSIFiBo toolbox. Superimposed is the contribution to the overall RMS value of the vertical acceleration of the building modes, within a frequency 


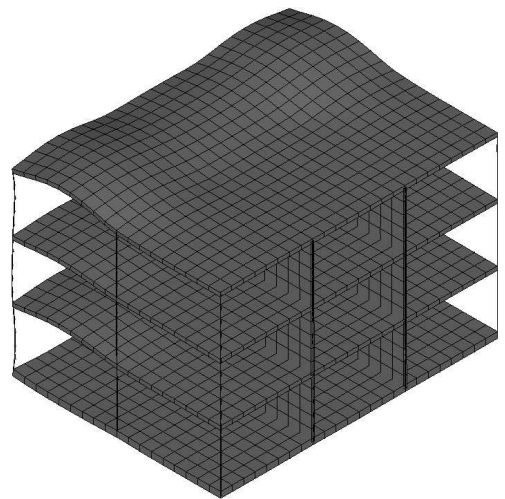

(a) Mode shape at $15.33 \mathrm{~Hz}$

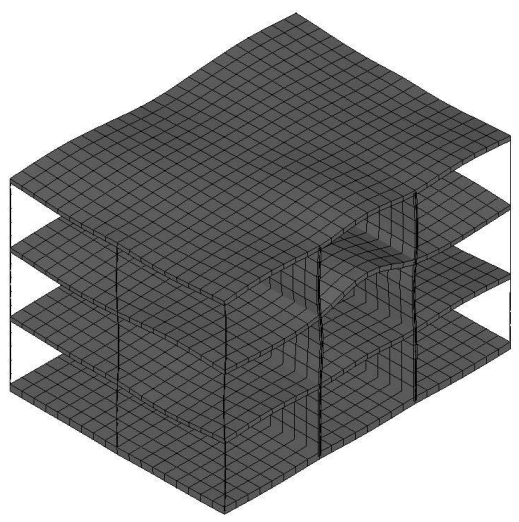

(d) Mode shape at $30.40 \mathrm{~Hz}$

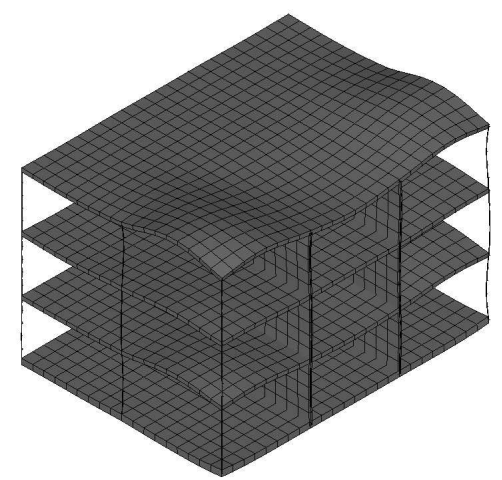

(b) Mode shape at $18.04 \mathrm{~Hz}$

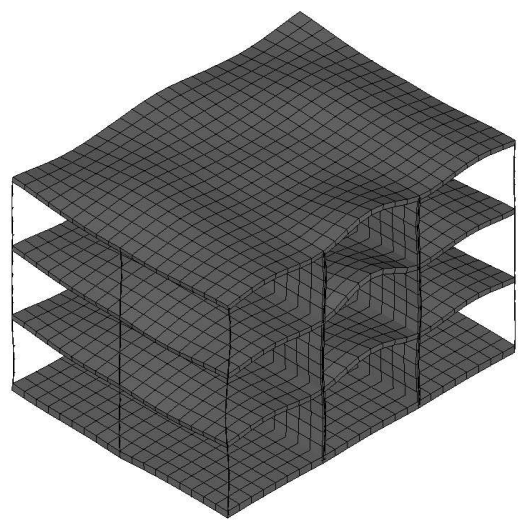

(e) Mode shape at $47.88 \mathrm{~Hz}$

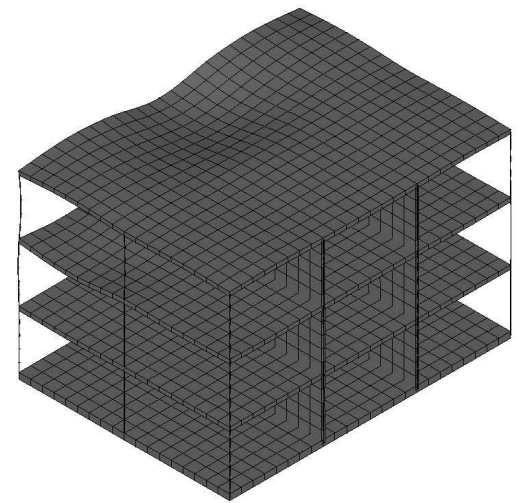

(c) Mode shape at $21.71 \mathrm{~Hz}$

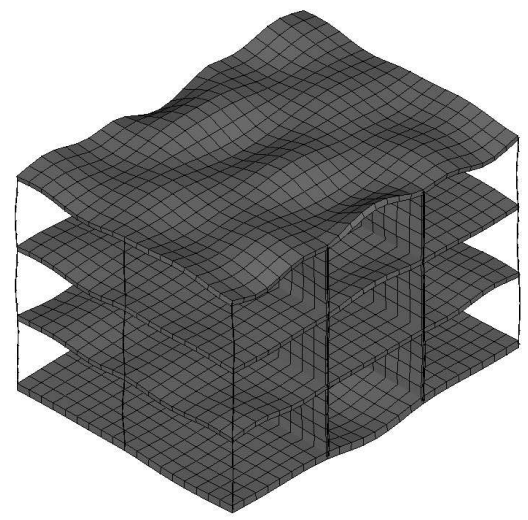

(f) Mode shape at $94.92 \mathrm{~Hz}$

Figure 2: Bending mode shapes of the floors.

band centred at $\Omega_{j}$, computed from the proposed scoping model as:

$$
C_{j}\left(\Omega_{j}\right)=\sum_{i} \sqrt{C_{i}^{2}\left(f_{i}\right)} \quad \forall f_{i} \in\left[\Omega_{j 0}, \Omega_{j 1}\right]
$$

where $\Omega_{j 0}$ and $\Omega_{j 1}$ are the limits of the one-third octave band $\Omega_{j}$, and $C_{i}$ is calculated from Equation (26).

The building response was evaluated at observation points $A, B, C, D, P$ and $W$ (Figure 1) located at every storey of the building. This response was mainly distributed in the frequency range from $8 \mathrm{~Hz}$ to $125 \mathrm{~Hz}$. The higher level of vibration was observed at the observation point $A$ (Figure 3. (a)) located at the part of the slabs supported on the core wall, where the bending stiffness of the floor was higher than in the remaining parts of the structure. The response at this point was found at frequencies of $30.40 \mathrm{~Hz}, 47.88 \mathrm{~Hz}$ and $94.92 \mathrm{~Hz}$ that correspond with those bending modes which present higher vertical displacements at the slabs bounded by the core wall (Figures 2.(d,e,f)). Conversely, the response in the slabs at observation points $B, C$ and $D$ (Figures 3.(b,c,d)) present lower value of vibration, and peaks (found at frequencies of 
$15.33 \mathrm{~Hz}$ and $18.04 \mathrm{~Hz}$ ) match the two first bending vertical modes (Figures 2.(a,b)). This is because the higher slab flexibility at these points causes an increased absorption of strain energy. The responses in the column (Figure 3.(e)) and the core wall (Figure 3.(f)) are distributed at approximately $47.88 \mathrm{~Hz}$. It should be mentioned that the observation point $W$ presents the lowest values of vibration. The agreement between the proposed scoping model and the SSIFiBo toolbox is good in the frequency range from 15 to $100 \mathrm{~Hz}$.

The overall RMS value of the acceleration response computed using both the proposed scoping model (Equation (24)), the SSIFiBo toolbox, and Equation (14) are shown in Figure 4. The discrepancies in the results obtained using Equation (14) and those computed without these simplifications are within a reasonable range of uncertainty, with the results obtained using Equation (24) being more accurate. The solution computed using the SSIFiBo toolbox shows a correlation between the building vibration and the storey level. However, this trend is not clearly observed in the scoping model solution. The differences between both models reaches the highest value in the first floor. Nevertheless, the uncertainties are below $13 \mathrm{~dB}$.

\subsection{Parametric study: soil properties and type of foundation}

Secondly, for the purpose of determining the versatility of the model, the influence of soil properties and building design on structural response was studied. Three types of soil with the properties summarized in Table 1 and five types of building were analysed. Each was similar to the generic building described in subsection 3.1, but with the following changes:

1. Foundation consisting of a slab with a thickness of $0.3 \mathrm{~m}$ as in subsection 3.1 .

2. Foundation consisting of a slab with a thickness of $0.5 \mathrm{~m}$.

3. Isolated footing of size $1.2 \mathrm{~m} \times 1.2 \mathrm{~m} \times 0.5 \mathrm{~m}$.

4. Continuous footing of size $1.2 \mathrm{~m} \times 0.5 \mathrm{~m}$.

5. Absence of core wall.

Table 1: Soil properties.

\begin{tabular}{c|cccc}
\hline Soil type & $c_{p}[\mathrm{~m} / \mathrm{s}]$ & $c_{s}[\mathrm{~m} / \mathrm{s}]$ & $\xi[-]$ & $\rho\left[\mathrm{kg} / \mathrm{m}^{3}\right]$ \\
\hline Soft & 300 & 150 & 0.06 & 1750 \\
Medium & 400 & 200 & 0.06 & 1750 \\
Stiff & 600 & 300 & 0.06 & 1750 \\
\hline
\end{tabular}

Figure 5 shows the overall RMS value of the building response at the top floor depending on the soil properties. The increment of building vibration with increasing soil stiffness observed in the solution computed from SSIFiBo toolbox it because the energy dissipation of soft soils is higher than stiff soils. This observation is not presented in the scoping model solution. Moreover the discrepancies between both models 


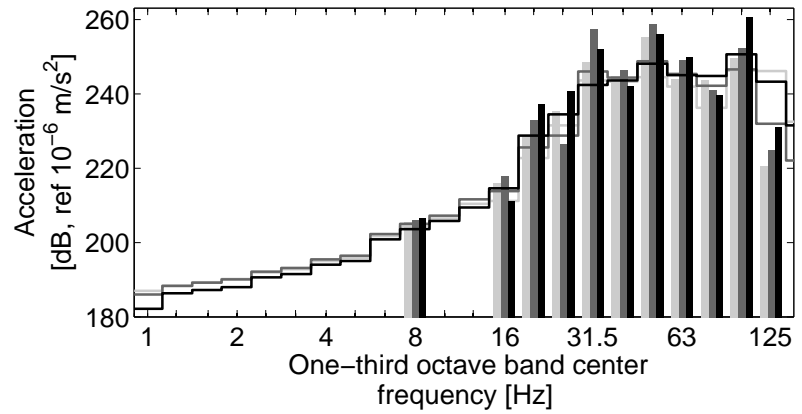

(a)

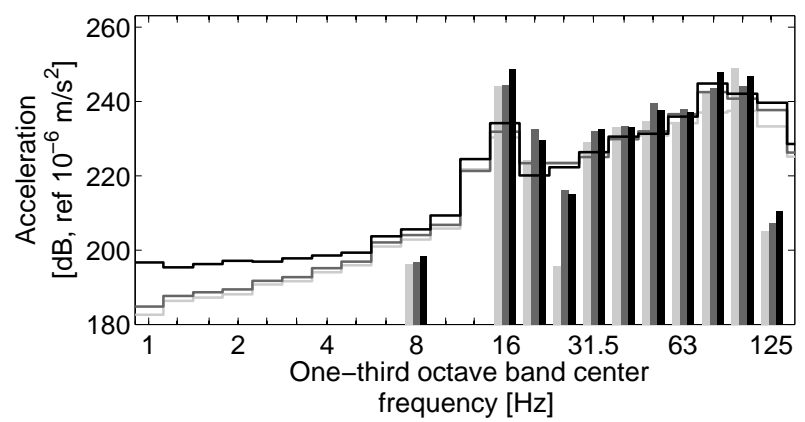

(c)

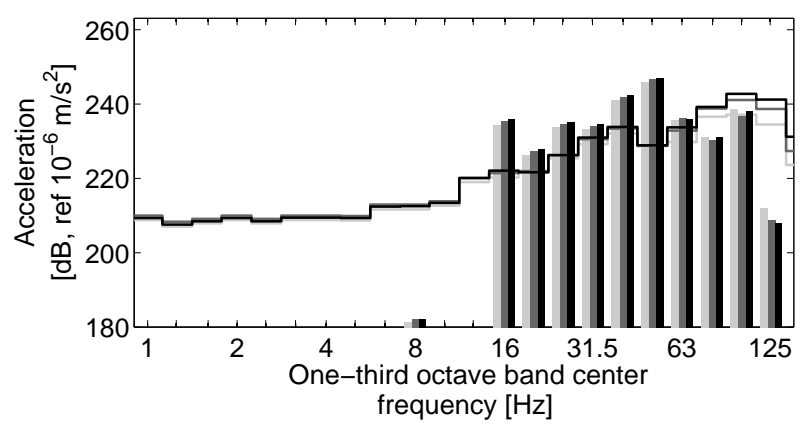

(e)

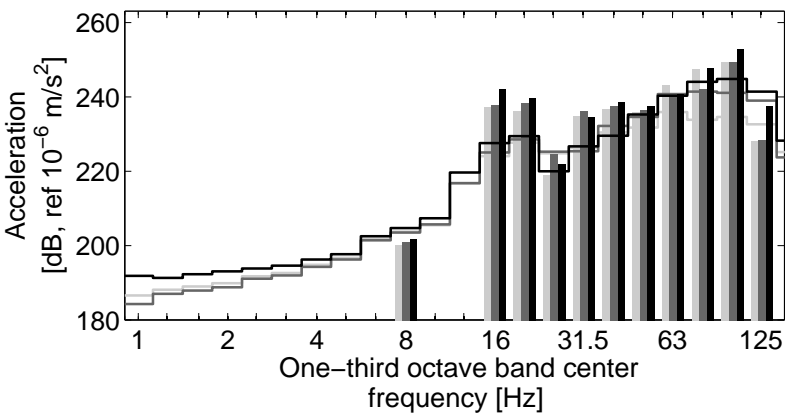

(b)

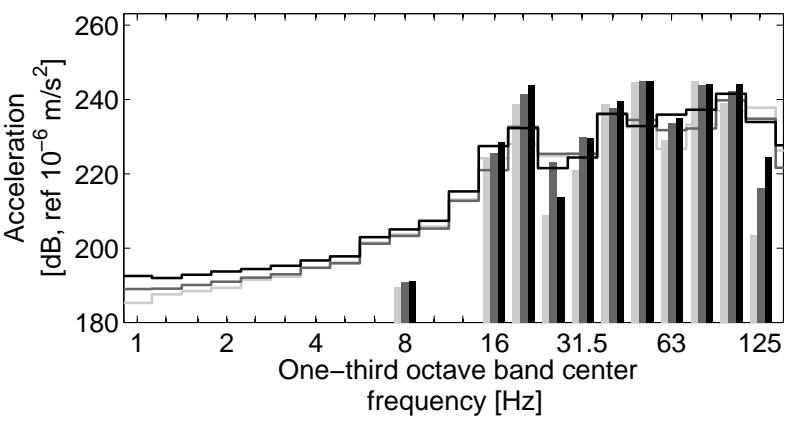

(d)

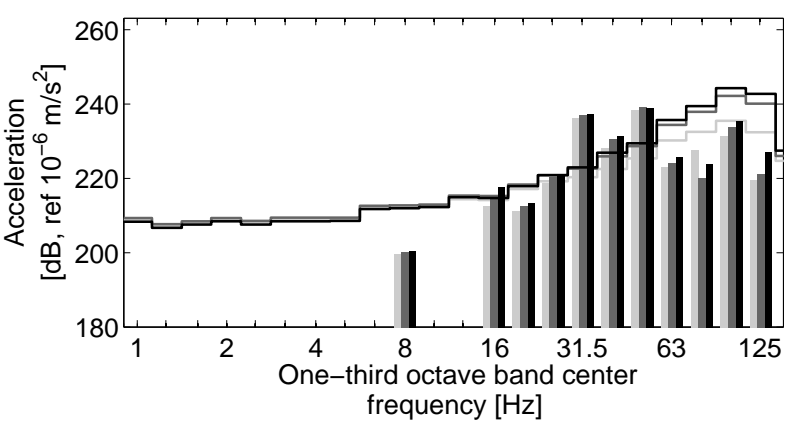

(f)

Figure 3: One-third octave band centre frequency of the vertical relative acceleration computed $\ddot{\mathbf{u}}(t)$ by the SSIFiBo toolbox [40] (solid lines) and contribution $C_{j}$ to the overall RMS value of the vertical acceleration of the modes within a frequency band centred in $\Omega_{j}$ obtained from the proposed scoping model (bars) at observation points (a) $A$, (b) $B$, (c) $C$, (d) $D$, (e) $P$ and (f) $W$ located at the first (light grey color), the second (dark grey color) and the third (black color) floors.

are higher in the soft soil. This is because the influence of SSI in the soft soil is dominant, and the scoping model uses a simplified calculation procedure in comparison to the SSIFiBo toolbox. In order to evaluate the uncertainty of the results, the simplifications assumed in Equation (14) and the methodology to evaluate the 


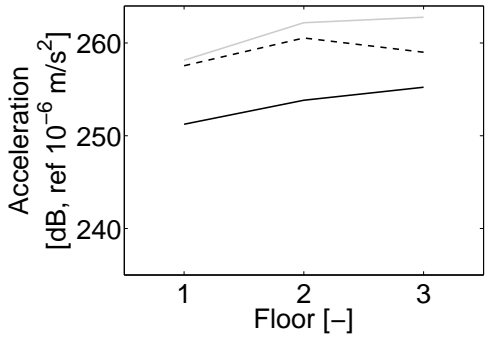

(a)

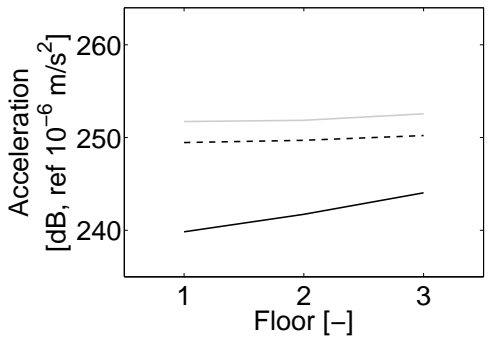

(d)

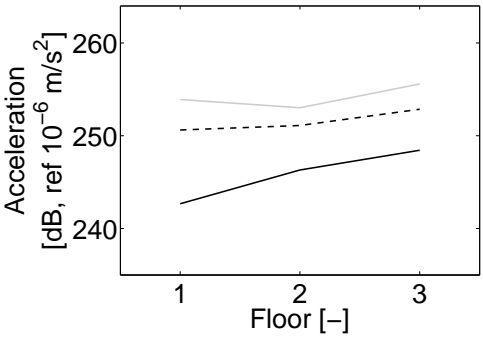

(b)

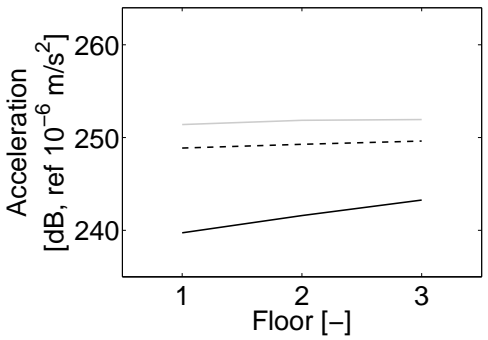

(e)

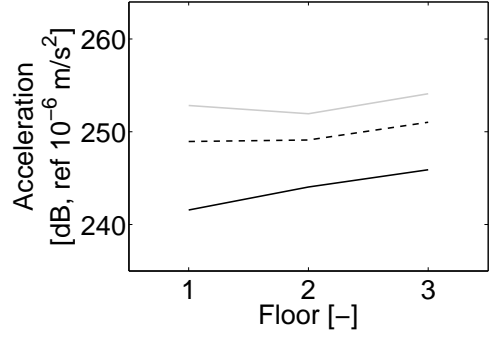

(c)

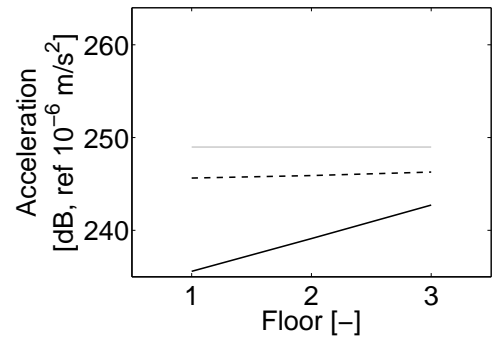

(f)

Figure 4: Overall RMS value of the acceleration response against the storey level at the observation points (a) $A$, (b) $B$, (c) $C$, (d) $D$, (e) $P$ and (f) $W$ computed from the SSIFiBo toolbox [40] (black solid line), the scoping model (grey solid line) and the proposed model without simplifications (black dashed line).

structural damping of the building in both models should be considered. Structural damping in the scoping model is determined using Equation (10), where the same damping $\zeta_{i}=\zeta$ for each $i$-th mode has been used. In comparison, the SSIFiBo toolbox considers viscous damping in the time domain, based on the Rayleigh model [32], and thus damping is not the same for all frequencies. In spite of this, the agreement between both models improves as soil stiffness increases and the uncertainty is within a reasonable range.

Regarding the analysis of different building parameters, Table 2 summarizes the obtained results. The influence of the thickness of the foundation slab causes only small changes to the building response as the thickness increased. Similar results were derived in previous researches [14, 41]. The isolated and continuous footing foundations yield lower values of acceleration than the $0.5 \mathrm{~m}$ thick slab showing that vibration levels decrease with decreasing the stiffness of the foundation. This phenomenon is because the $0.5 \mathrm{~m}$ thick slab is the stiffest foundation, and has lower energy dissipation capacity than the remaining foundations. In comparison, the use of a core wall in the building increases the structural stiffness and the level of the response.

Considering the accuracy of the scoping model for a wide range of different soils and building parameters, it was concluded that it is suitable for use in a wide range of scenarios. 


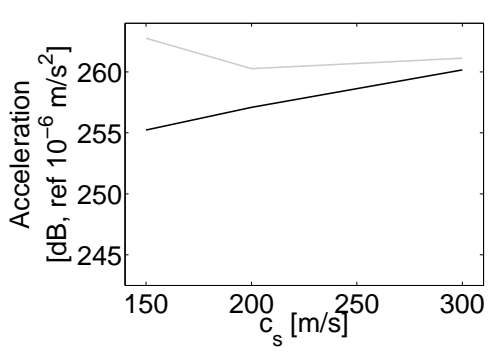

(a)

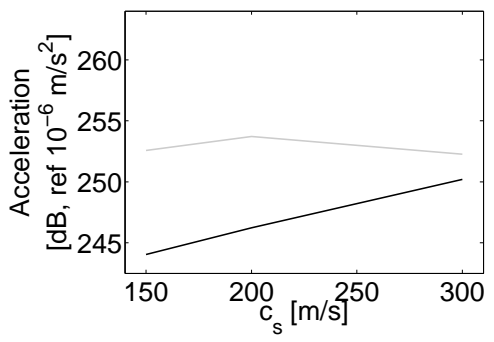

(d)

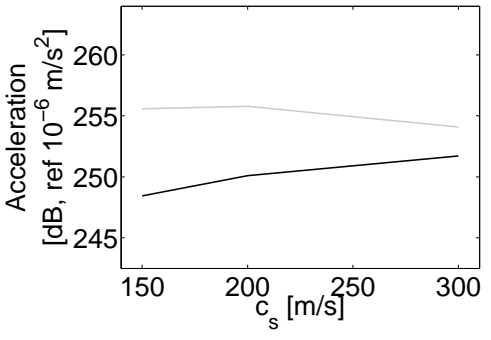

(b)

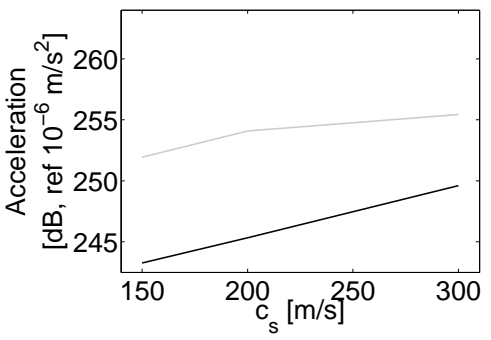

(e)

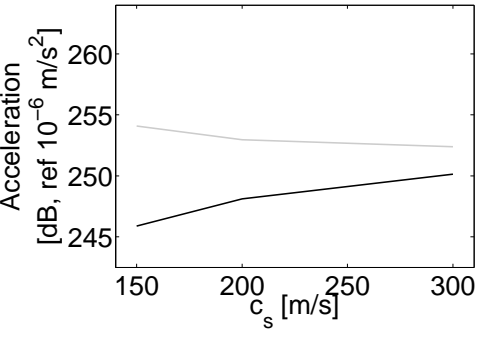

(c)

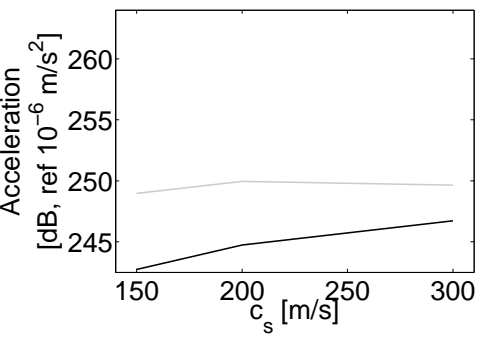

(f)

Figure 5: Overall RMS value of the acceleration response depending of the soil properties at the top floor at the observation points (a) $A$, (b) $B$, (c) $C$, (d) $D$, (e) $P$ and (f) $W$ computed from the SSIFiBo toolbox (black line) and the scoping model (Equation (24)) (grey line).

Table 2: Maximum of the overall RMS value of the acceleration response for each observation point.

\begin{tabular}{l|lccccccc}
\hline Problem & $\begin{array}{c}\text { Point } A \\
{[\mathrm{~dB}]}\end{array}$ & $\begin{array}{c}\text { Point } B \\
{[\mathrm{~dB}]}\end{array}$ & $\begin{array}{c}\text { Point } C \\
{[\mathrm{~dB}]}\end{array}$ & $\begin{array}{c}\text { Point } D \\
{[\mathrm{~dB}]}\end{array}$ & $\begin{array}{c}\text { Point } P \\
{[\mathrm{~dB}]}\end{array}$ & $\begin{array}{c}\text { Point } W \\
{[\mathrm{~dB}]}\end{array}$ & $\begin{array}{c}\text { Maximum difference } \\
{[\mathrm{dB}]}\end{array}$ \\
\hline & Slab thickness $d=0.3 \mathrm{~m}$ & 262.8 & 255.6 & 254.1 & 252.6 & 251.9 & 248.9 & 10.8 \\
& Slab thickness $d=0.5 \mathrm{~m}$ & 264 & 255.7 & 256.2 & 254.8 & 254.8 & 249.9 & 11.5 \\
Isolated footing & 259.1 & 252.5 & 252.4 & 250.9 & 251.9 & 248.4 & 11.5 \\
Continuous footing & 259.6 & 255.2 & 254.4 & 255.1 & 254.9 & 248.6 & 11.4 \\
Without core wall & 256.1 & 254.7 & 255.1 & 255.1 & 249.9 & 251.8 & 9.1 \\
\hline
\end{tabular}

\section{Sensitivity analysis of building induced vibration due to train passage}

In this section, vibrations induced by train passages in three multi-storey buildings are evaluated using the scoping model. The influence of soil properties, building height, train speed and the distance from the track to the building on the results are analysed. The midpoint foundation of the building was located at distances, $\{20,30,40,50,60,70\} \mathrm{m}$ from the track centreline and three different homogeneous soils were considered with the properties indicated in Table 3. Table 4 shows the carriage length $L_{t}$, the distance between bogies $L_{b}$, the axle distance $L_{a}$, the total axle mass $M_{t}$ and the unsprung axle mass $M_{u}$ for all carriages of the S-100 serie train considered in this paper. Train speeds of $\{100,150,200\} \mathrm{km} / \mathrm{h}$ were 
analysed. In all cases, train speed was found to be in a range between $10 \%$ and $60 \%$ of the critical velocity of the track system [42]. Therefore, it was assumed that the dynamic contribution (e.g. rail unevenness) would be dominant in the free-field response [6]. In total, the sensitivity study included the analysis of 162 problems ( 3 soil types $\times 3$ buildings $\times 3$ train speeds $\times 6$ distances $)$.

Table 3: Soil properties.

\begin{tabular}{c|cccc}
\hline Soil type & $c_{p}[\mathrm{~m} / \mathrm{s}]$ & $c_{s}[\mathrm{~m} / \mathrm{s}]$ & $\xi[-]$ & $\rho\left[\mathrm{kg} / \mathrm{m}^{3}\right]$ \\
\hline Soft & 250 & 100 & 0.06 & 1750 \\
Medium & 400 & 200 & 0.06 & 1800 \\
Stiff & 995 & 300 & 0.06 & 1850 \\
\hline
\end{tabular}

Table 4: Geometrical and mass characteristics of the S-100 train.

\begin{tabular}{c|lccccccc}
\hline & & No. of carriages & No. of axles & $L_{t}[\mathrm{~m}]$ & $L_{b}[\mathrm{~m}]$ & $L_{a}[\mathrm{~m}]$ & $M_{t}[\mathrm{~kg}]$ & $M_{u}[\mathrm{~kg}]$ \\
\hline \multirow{2}{*}{} & Traction cars & 2 & 4 & 22.15 & 14.00 & 3.00 & 17185 & 2048 \\
í & End carriages & 2 & 3 & 21.84 & 18.70 & 3.00 & 11523 & 2003 \\
& Central carriages & 6 & 2 & 18.70 & 18.70 & 3.00 & 15523 & 2003 \\
\hline
\end{tabular}

The structures were four, eight and twelve storeys buildings with the same floor plan dimensions $12 \mathrm{~m} \times$ $12 \mathrm{~m}$ (Figure 6.(a)). It consisted of eight concrete columns with $0.6 \mathrm{~m} \times 0.4 \mathrm{~m}$ section, four edge beams with $0.6 \mathrm{~m} \times 0.2 \mathrm{~m}$ section and two framed concrete walls with $2.4 \mathrm{~m} \times 0.15 \mathrm{~m}$ section. The floors were simply supported concrete slabs with a thickness of $0.2 \mathrm{~m}$. The floors consist of a two-dimensional frame with axial stiffness per unit length $E A=1.433 \times 10^{9} \mathrm{~N} / \mathrm{m}$, bending stiffness per unit length $E I=9.935 \times 10^{6} \mathrm{Nm}$, and a mass per unit area of $m=172 \mathrm{~kg} / \mathrm{m}^{2}$. The structure was founded on a $1.0 \mathrm{~m}$ thick concrete slab. The concrete material had the following properties: Young's modulus $E=20 \times 10^{9} \mathrm{~N} / \mathrm{m}^{2}$, Poisson's ratio $\nu=0.2$, density $\rho=2400 \mathrm{~kg} / \mathrm{m}^{3}$ and the structural damping was considered using a Rayleigh model, where $\zeta=0.05$ was set for all modes that contributed to the building response. The structure was discretised using two-node Euler-Bernoulli elements to represent columns and beams and four-node shell elements for the floors and the framed walls. Figure 6.(b) shows the discretisation of the twelve-storey building.

The bending mode shapes of the floors computed without considering SSI are presented in Figure 7 . The mode shapes can be observed for increasing core wall (Figures 7.(e,j,n)) and corner column (Figures 7. $(\mathrm{d}, \mathrm{g}, \mathrm{l}, \mathrm{m}, \mathrm{o}))$ deformations. Regarding the core wall, the displacements at the central zone of the floors are larger, while the corner columns involve the bending of the columns.

In the next subsection, before the sensitivity analysis, the dynamic behaviour of the buildings considering SSI will be studied.

The soil vibrations due to train passages were numerically obtained using the SSIFiBo toolbox [40]. The rails were represented by Euler-Bernoulli beams with a bending stiffness $E_{\mathrm{r}} I_{\mathrm{r}}=6.45 \times 10^{6} \mathrm{~N} / \mathrm{m}^{2}$ and a mass 


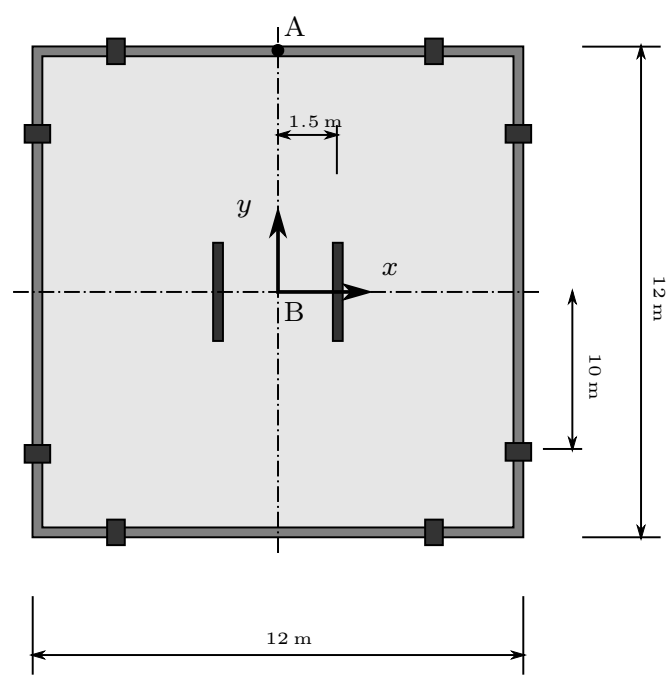

(a)

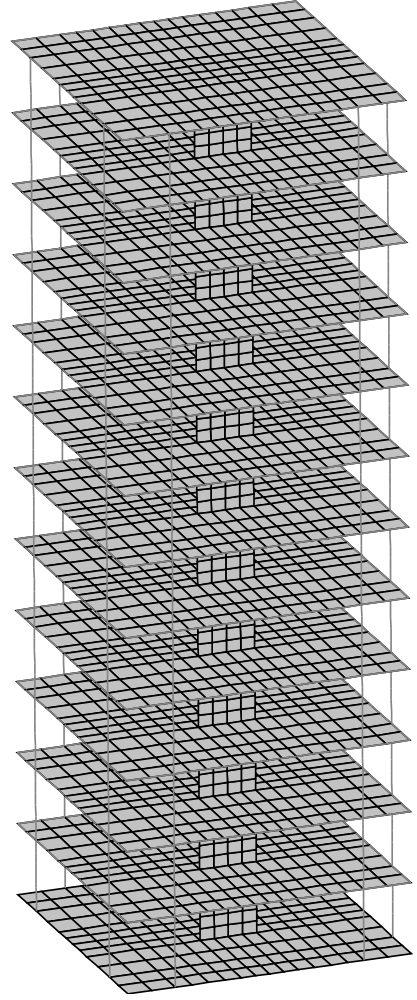

(b)

Figure 6: (a) Four, eight and twelve-storey buildings plan geometry and (b) discretization of the twelvestorey building.

per unit length $\rho_{\mathrm{r}} A_{\mathrm{r}}=60.34 \mathrm{~kg} / \mathrm{m}$ for each rail. The rail pads were modelled as continuous spring-damper connections. A rail pad stiffness $k_{\mathrm{rp}}=150 \times 10^{6} \mathrm{~N} / \mathrm{m}$ and loss factor $\eta_{\mathrm{rp}}=0.25$ to account for internal energy dissipation in the rail pad were used. The sleepers were of concrete monoblock type with a spacing of $d=0.60 \mathrm{~m}$ and modelled as a uniformly distributed mass being $m_{\mathrm{sl}}=300 \mathrm{~kg}$. The ballast bed was represented by a set of distributed linear springs and dampers. A ballast layer with a thickness $h_{\mathrm{b}}=0.35 \mathrm{~m}$, vertical stiffness $k_{\mathrm{b}}=500 \times 10^{6} \mathrm{~N} / \mathrm{m}$ and density $\rho_{b}=1550 \mathrm{~kg} / \mathrm{m}^{3}$ was considered.

In the free-field predictions, both quasi-static excitation and dynamic excitation due to random track unevenness were taken into account [6]. The same track unevenness profile was considered for all the cases.

Once the free-field vibration was computed, ground-borne response spectra $\Lambda_{i}^{j}\left(f_{i}\right)$ for a damping ratio $\zeta=0.05$ was obtained using Equation (25). Then, the building response was evaluated. The building response was obtained using a single point response (SPR) excitation model, where the incident wave was transmitted simultaneously to all nodes of the structure foundation. The considered tolerance $(\varepsilon=0.01)$ was small enough to ensure that the building behaviour was accurately obtained. The building responses at 


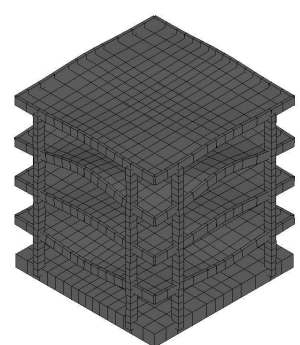

(a) Mode at $13.93 \mathrm{~Hz}$

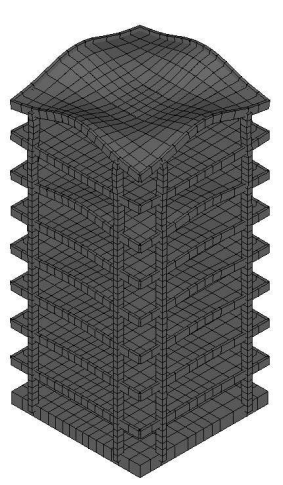

(f) Mode at $11.46 \mathrm{~Hz}$

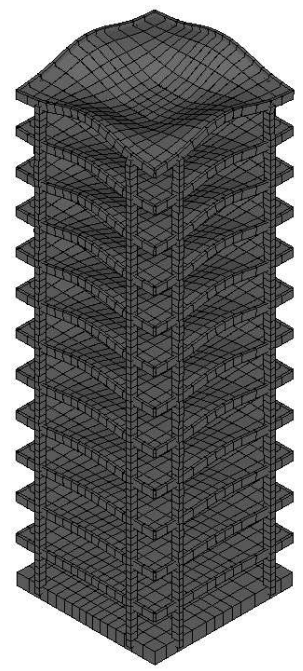

(k) Mode at $9.10 \mathrm{~Hz}$

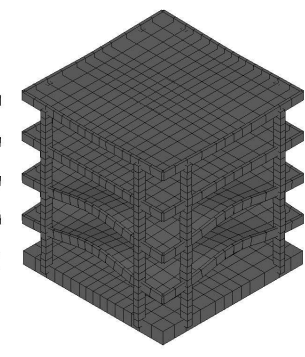

(b) Mode at $14.25 \mathrm{~Hz}$

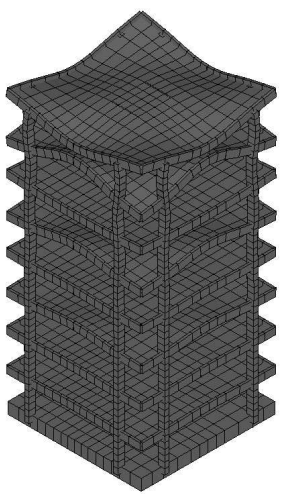

(g) Mode at $12.35 \mathrm{~Hz}$
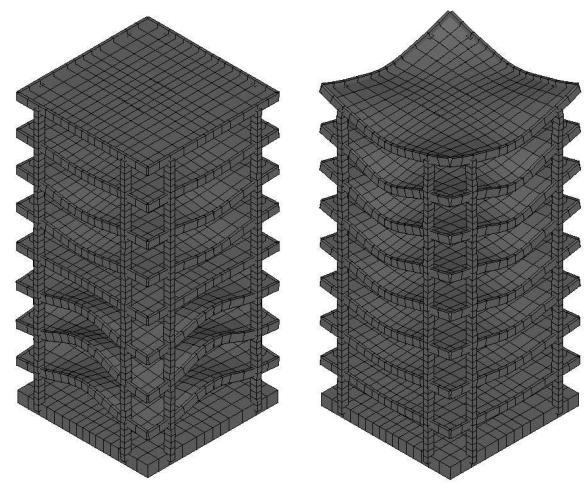

(i) Mode at $20.67 \mathrm{~Hz}$
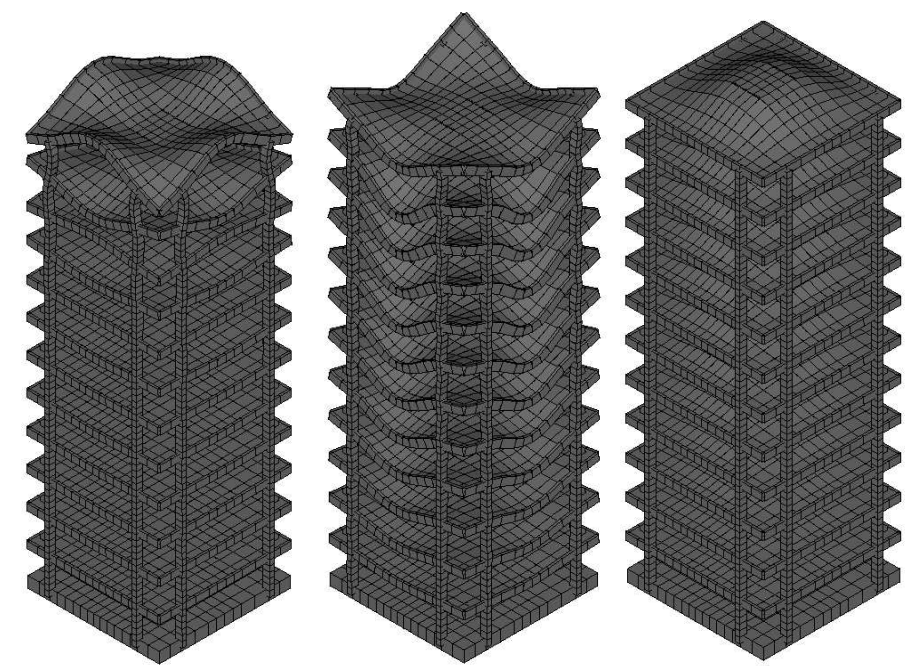

(n) Mode at $20.14 \mathrm{~Hz}$ (j) Mode at $30.19 \mathrm{~Hz}$

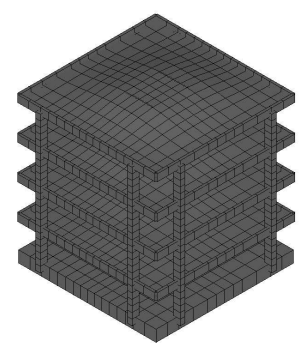

(e) Mode at $60.38 \mathrm{~Hz}$
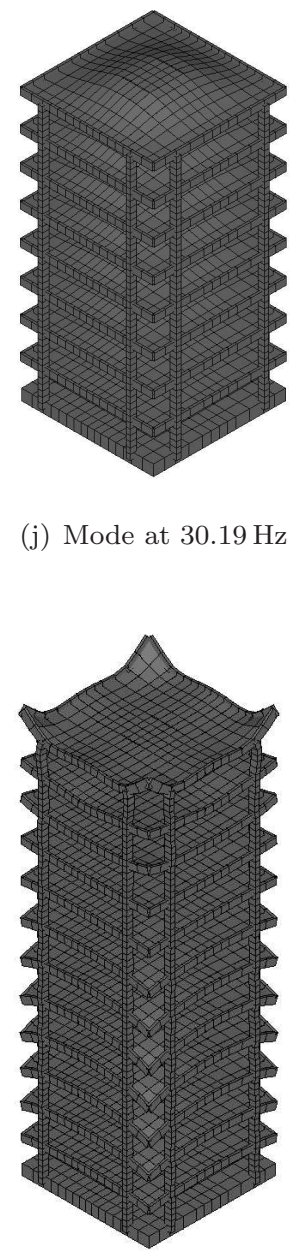

(o) Mode at $30.21 \mathrm{~Hz}$

Figure 7: Bending floor mode shapes of the (a,b,c,d,e) four-storey building, (f,g,h,i,j) eight-storey building and $(\mathrm{k}, \mathrm{l}, \mathrm{m}, \mathrm{n}, \mathrm{o})$ twelve-storey building.

the points $A$ and $B$ (Figure 6.(a)) located along all the storey levels were analysed.

In this sensitivity analysis, the results from the scoping model were compared with those obtained by 
the SSIFiBo toolbox [40].

\subsection{Soil properties}

Before the sensitivity analysis, building response was characterized depending on the soil properties. For this purpose, the contribution of bending modes was obtained from the building response to an incident wave field acting in the vertical $(z)$ direction using a ground-borne response spectra $\Lambda_{i}^{j}\left(f_{n}\right)=1 \mathrm{~m} / \mathrm{s}^{2}$ (Equation 24). In this way, the contribution of each $i$-th mode to the building response $C_{i}=\sqrt{\phi_{i}^{2} \sum_{j=1}^{3} \Gamma_{i}^{j^{2}}}$ (Equation (26)) was not dependent of the excitation. Figure 8 shows the contribution to the overall RMS value of the vertical acceleration at the different frequencies for the four, eight and twelve storey buildings obtained using Equation (28) and evaluated at the top floor, at observation points $A$ and $B$ (Figure 6.(a)). The response was computed for the soils presented in Table 3. The bending modes of the dominant floors were found in the frequency range below $80 \mathrm{~Hz}$. It was observed that the contribution for each soil was different. At the observation point $B$, the fundamental frequency was different depending on the soil properties.

Next, the combination of the response spectra $\Lambda_{i}^{j}$ (computed from the free-field predictions) and the characterization of the buildings for a load with constant amplitude at the studied frequency range (Figure 8 ) is used to understand the building behaviour due to train passages. The effect of the soil properties on the scoping prediction for a building located at $20 \mathrm{~m}$ from the track due to a S-100 train travelling at $v=150 \mathrm{~km} / \mathrm{h}$ was studied. Figure 9 shows the ground-borne response spectra $\Lambda_{i}^{j}$ computed from the free field vibrations. The ground-borne spectra exhibits elevated amplitudes in the frequency range between $10 \mathrm{~Hz}$ and $40 \mathrm{~Hz}$. Peaks around the axle passing frequency $f_{a}=v / L_{a}=13.9 \mathrm{~Hz}$ and due to the dynamic excitation at $30 \mathrm{~Hz}$ can be observed. The highest value was reached in the vertical direction. The effect of the soil properties on the quasi-static contribution are clearly observed at lower frequencies. However, at the frequencies contributing to the dynamic response, the results do not show a clear correlation between the free field response and the soil properties.

Figure 10 shows the influence of the soil on building vibration. It can be observed that the accelerations decrease as the soil stiffness increases, excluding the eight-storey building where the response in the medium soil is higher. This phenomenon can be explained from $\Lambda_{i}^{j}$ (Figure 9) and the eight-storey building response showed in Figure 8. Ground-borne vertical response spectra $\Lambda_{i}^{j}$ (Figure 9.(c)) shows higher amplitudes in the medium soil at about $30 \mathrm{~Hz}$ because this is close to the the fundamental frequency for the observation point $B$ (Figure 8. (d)). In Figure 8.(c) it is observed that the response at point $A$ is concentrated around $10 \mathrm{~Hz}$ and the vibration level in the stiff soil is slightly higher than for the medium soil. However, the excitation $\Lambda_{i}^{j}$ around $10 \mathrm{~Hz}$ (Figure 9.(c)) presents a lower value for the stiff soil. Thus, the eight-storey building responses at point $A$ for both the medium and the stiff soil are similar (Figure 10. (b)).

The scoping model predicted higher amplitudes than the SSIFiBo reference model. The differences between both models were dependent on the soil properties, but these uncertainties did not follow a clear 


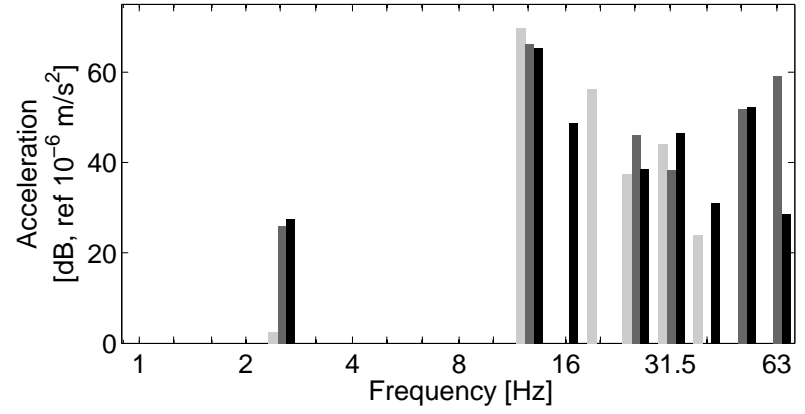

(a)

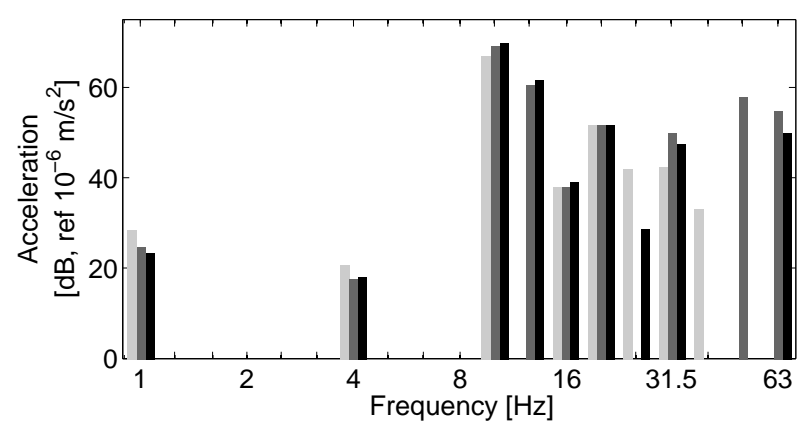

(c)

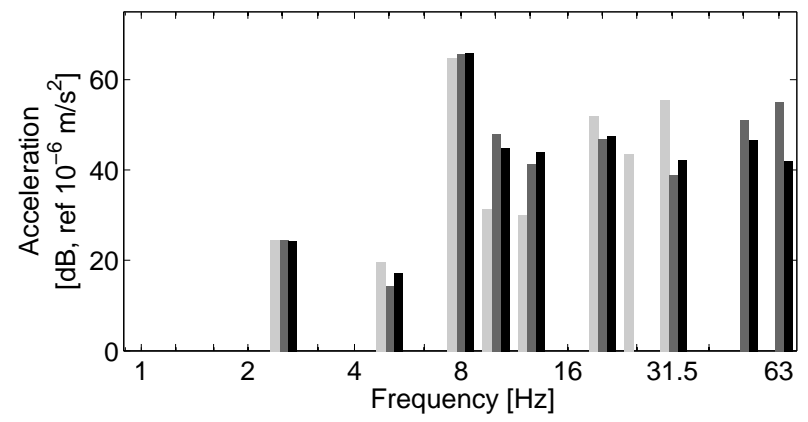

(e)

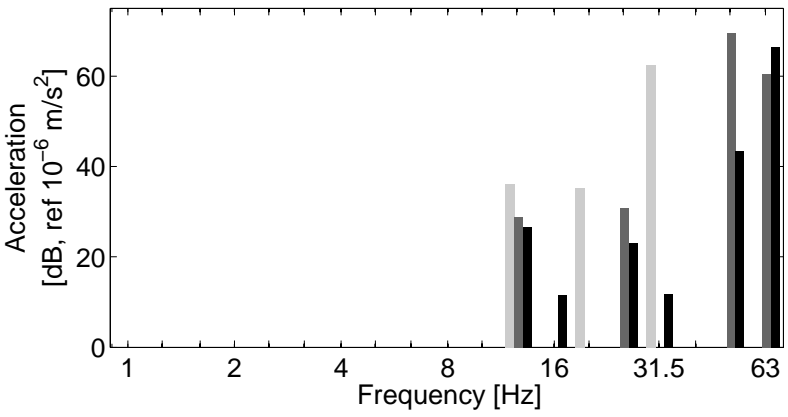

(b)

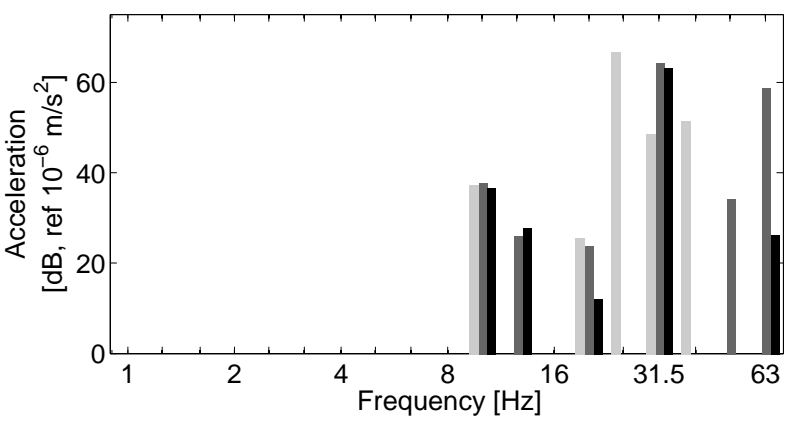

(d)

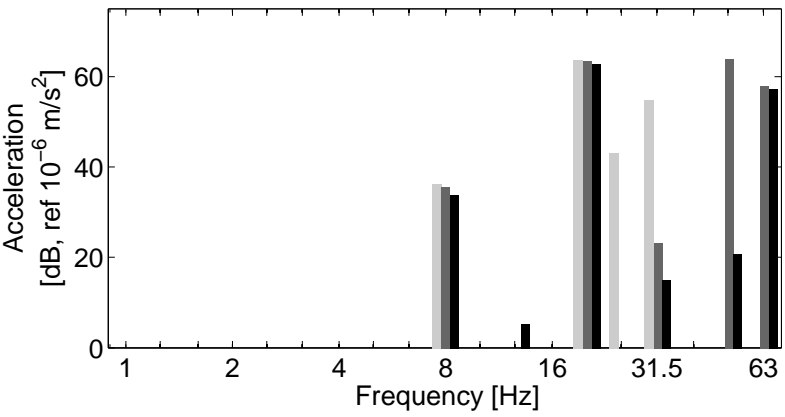

(f)

Figure 8: Contribution of the modes to the overall RMS value of the vertical acceleration due to an incident wave with ground-borne response spectra $\Lambda_{i}^{j}=1 \mathrm{~m} / \mathrm{s}^{2}$ in the soft soil (light grey bar), medium soil (dark grey bar) and stiff soil (black bar) obtained from the proposed scoping model at the top floor of the observation points (a,c,e) $A$ and (b,d,f) $B$ for the (a,b) four-storey building, (c,d) eight-storey building and (e,f) twelvestorey building.

trend. Thus, it can be concluded that soil properties are an important parameter for the accuracy of the proposed scoping model. 


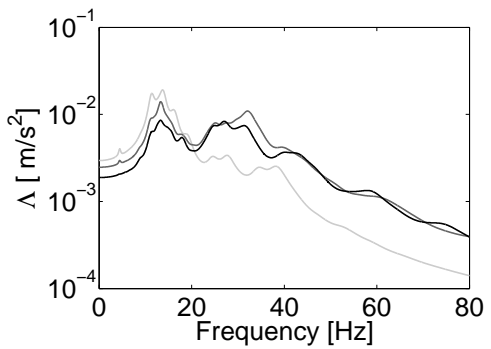

(a)

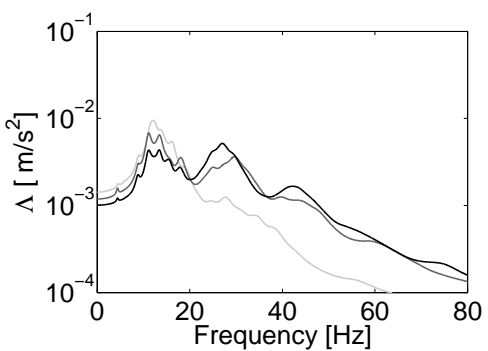

(b)

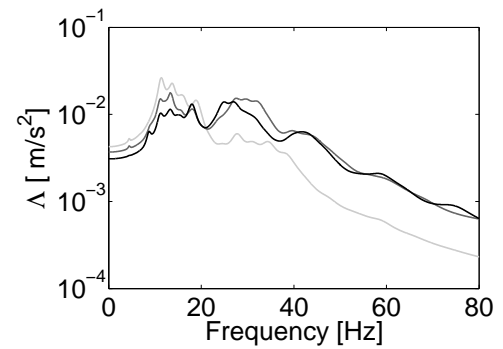

(c)

Figure 9: (a) Transversal, (b) longitudinal and (c) vertical ground-borne response spectra $\Lambda_{i}^{j}$ at $20 \mathrm{~m}$ from the track center due to a train passage at $v=150 \mathrm{~km} / \mathrm{h}$ in the soft soil (light grey line), medium soil (dark grey line) and stiff soil (black line).

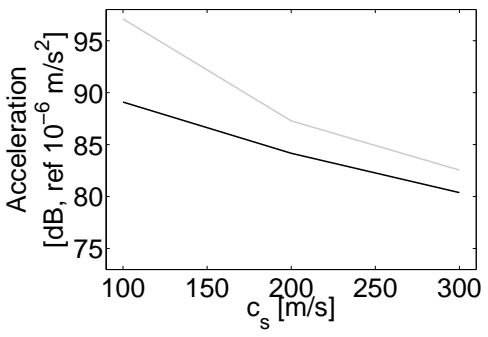

(a)

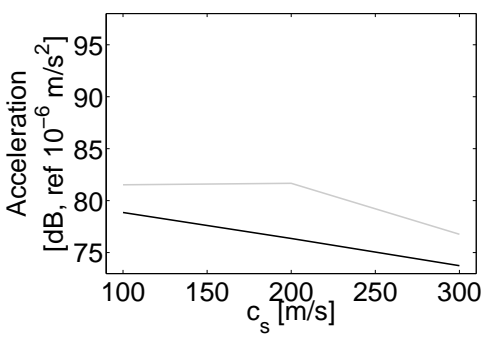

(d)

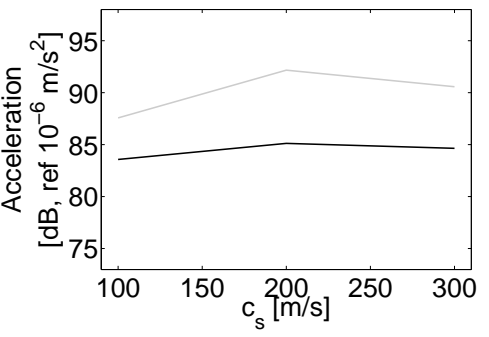

(b)

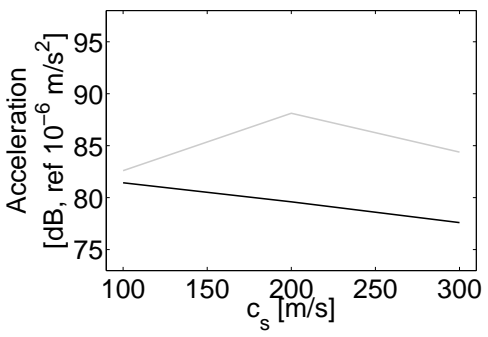

(e)

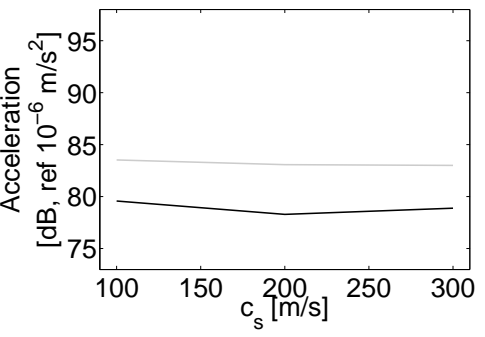

(c)

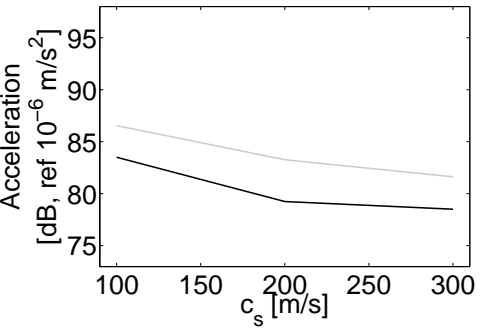

(f)

Figure 10: Overall RMS value of the acceleration response due to a train passage at $v=150 \mathrm{~km} / \mathrm{h}$ evaluated at the top floor at the observation points (a,b,c) $A$ and (d,e,f) $B$ computed from the SSIFiBo toolbox (black line) and the scoping model (Equation (24)) (grey line) for the (a,d) four-storey, (b,e) eight-story and (c,f) twelve-storey buildings.

\subsection{Building height}

Next, the effect of the building height on the results computed from the proposed methodology was analysed. The four, eight and twelve storey building responses due to the passage of a S-100 train travelling at $v=150 \mathrm{~km} / \mathrm{h}$ was analysed. The buildings were located at $20 \mathrm{~m}$ from the track and the soil with 
$c_{s}=200 \mathrm{~m} / \mathrm{s}$ was considered. Figure 11 shows the one-third octave band spectra content of the vertical relative accelerations $\ddot{\mathbf{u}}(t)$ (Equation (2)) at the observation points $A$ and $B$ located at the first, middle and top floors obtained from the SSIFiBo toolbox. Superimposed is the contribution to the overall RMS value of the frequencies computed from the scoping model. It can be seen that the mode with higher participation factor computed for the four, eight and twelve-storey buildings was found around the frequencies $12 \mathrm{~Hz}, 10 \mathrm{~Hz}$ and $8 \mathrm{~Hz}$ for the observation point $A$, and $50 \mathrm{~Hz}, 30 \mathrm{~Hz}$ and $20 \mathrm{~Hz}$ for the observation point $B$ respectively. Thus, as expected, frequency reduced as the building height increased. The highest value of vibration was found at the top floor of the eight-storey building because the values of the soil response spectra $\Lambda_{i}^{j}$ (Figure 9.(c)) match with the natural frequencies of the eight-storey building at about $10 \mathrm{~Hz}$ and $30 \mathrm{~Hz}$ (Figure 8. (c,d)). These are higher than those at the frequencies $8 \mathrm{~Hz}$ and $20 \mathrm{~Hz}$ that correspond to the natural frequencies of the twelve-storey building (Figures 8.(e,f)). The agreement between both models was quite good at the frequencies that dominate the building response.

Figure 12 presents the influence of building height on the overall RMS value of the response. The results computed from both models are shown for different storey levels. As expected, the response increases with storey level at the observation point $B$. However, this correlation is not observed at observation point $A$ for the four and twelve-storey buildings. Regarding the response at the observation point $A$ of the four-storey building, the response is at about $12 \mathrm{~Hz}$ that corresponds with the two first bending modes (Figure 7. (a,b)). These modes present larger amplitudes at the middle floors of the building than at the top floor. The lack of correlation between storey level and the response computed from the SSIFiBo toolbox at the observation point $A$ of the twelve-storey building can be explained since the second bending mode at $12 \mathrm{~Hz}$ (Figure 7.(1)) presents lower amplitudes at the floors from one to six at observation point $A$. The maximum discrepancy between both models was found in the response of the eight-storey building, where a difference of $8.5 \mathrm{~dB}$ was found. This discrepancy is acceptable considering the simplified procedure used to formulate the scoping model, and the different structural damping approaches used for it compared to the detailed model.

\subsection{Train Speed}

Next the scoping model was used to assess the effect of the train speed on building response. The response of the three buildings located at $20 \mathrm{~m}$ to the track due to the passage of a S-100 train travelling at $\{100,150,200\} \mathrm{km} / \mathrm{h}$ was studied. The moderately stiff soil was again considered. Figure 13 shows the vertical ground-borne response spectra $\Lambda_{i}^{j}$ computed from the free-field vibrations. Peaks around the axle passing frequency $f_{a}=v / L_{a}=\{9.26,13.9,18.52\} \mathrm{Hz}$ that involve the quasi-static contribution can be observed. The highest value was found in the ground-borne response spectra at $v=200 \mathrm{~km} / \mathrm{h}$ around $18 \mathrm{~Hz}$. The ground-borne response spectra due to a train passage at $v=150 \mathrm{~km} / \mathrm{h}$ resulted in peaks around $13 \mathrm{~Hz}$ and $30 \mathrm{~Hz}$.

The overall RMS value of the building response is shown in Figure 14. It can be seen that the level 


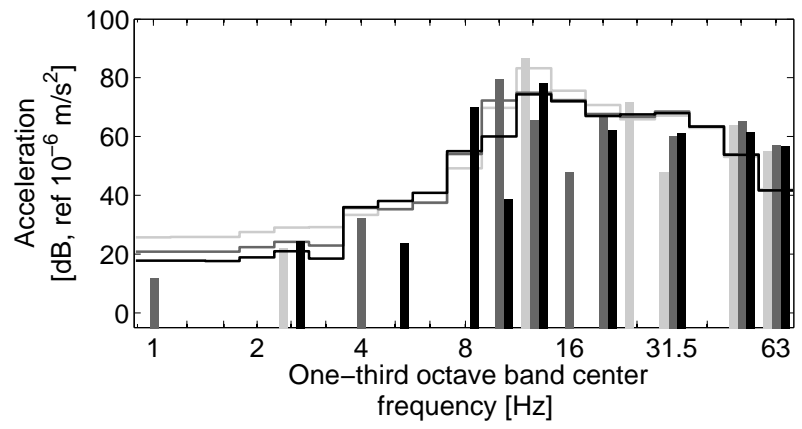

(a)

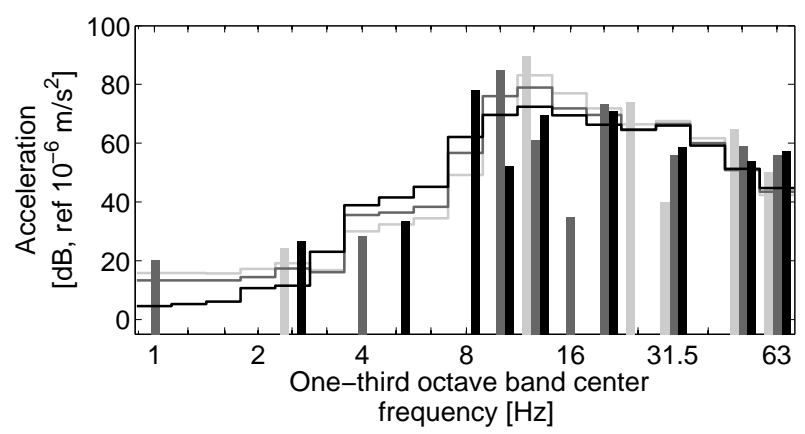

(c)

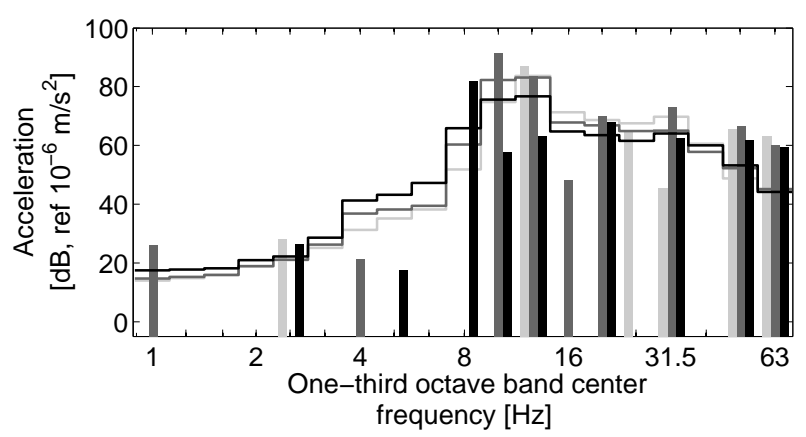

(e)

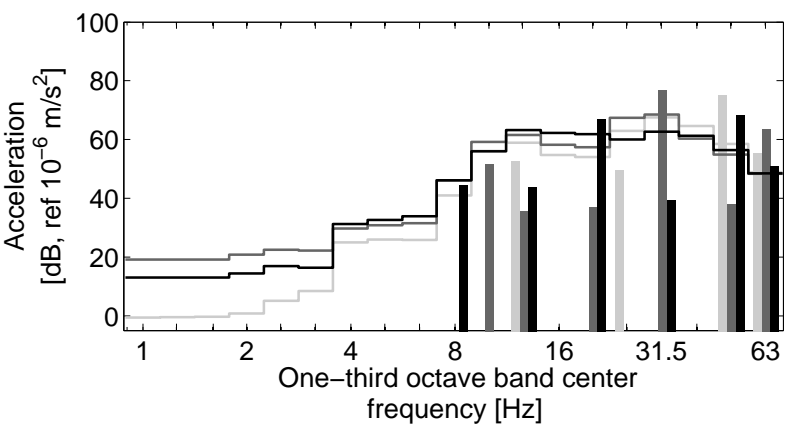

(b)

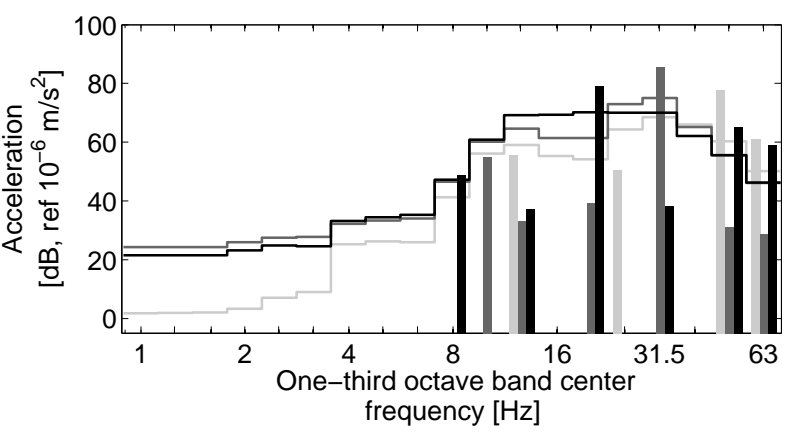

(d)

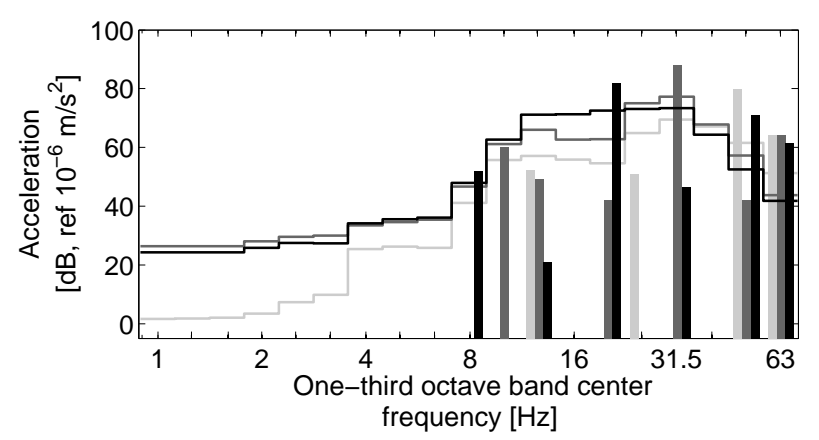

(f)

Figure 11: One-third octave band centre frequency content of the vertical relative acceleration due to a train passage at $v=150 \mathrm{~km} / \mathrm{h}$ computed by the SSIFiBo toolbox (lines) and contribution of the modes to the overall RMS value of the vertical acceleration obtained from the scoping model (bars) at the observation points (a,c,e) $A$ and $(\mathrm{b}, \mathrm{d}, \mathrm{f}) B$ located at $(\mathrm{a}, \mathrm{b})$ the first, $(\mathrm{c}, \mathrm{d})$ the middle and $(\mathrm{e}, \mathrm{f})$ the top floors of the four-storey building (light grey color), eight-storey building (dark grey color) and twelve-storey building (black color).

of vibration generally increased with increasing speed, except for the response at the observation point $B$ of the eight-storey building, computed by the scoping model. Instead, at approximately $30 \mathrm{~Hz}$ the ground- 


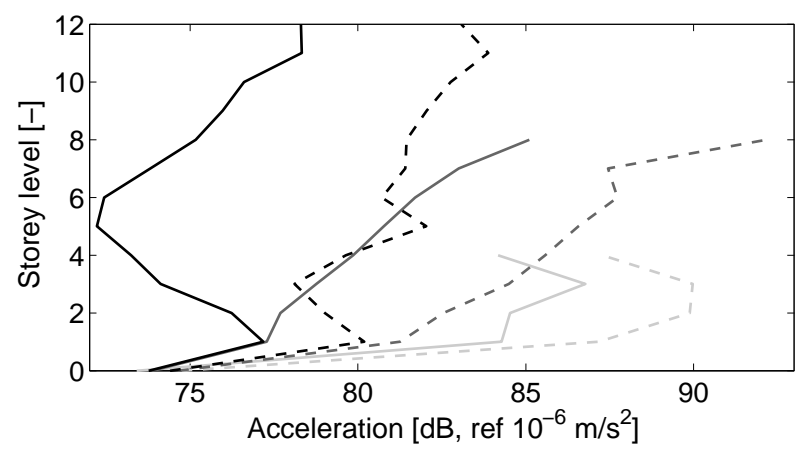

(a)

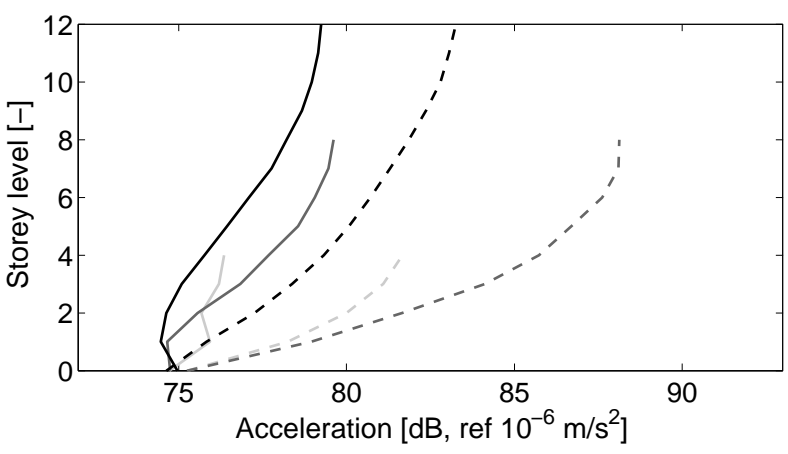

(b)

Figure 12: Overall RMS value of the acceleration response due to a train passage at $v=150 \mathrm{~km} / \mathrm{h}$ evaluated at the observation points (a) $A$ and (b) $B$ computed from the SSIFiBo toolbox (solid line) and the scoping model (Equation (24)) (dashed line) for the four-storey building (light grey line), eight-storey (dark grey line) and twelve-storey building(black line).

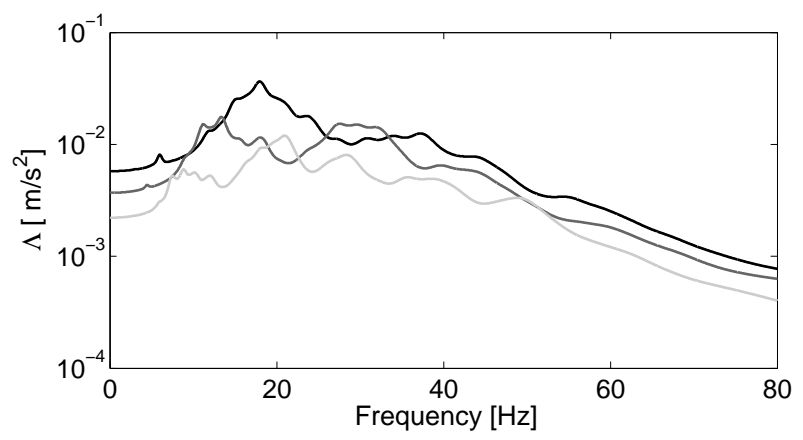

Figure 13: Vertical ground-borne response spectra $\Lambda_{i}^{j}$ at $20 \mathrm{~m}$ from the track center due to a train passage at $v=100 \mathrm{~km} / \mathrm{h}$ (light grey line), $v=150 \mathrm{~km} / \mathrm{h}$ (dark grey line) and $v=200 \mathrm{~km} / \mathrm{h}$ (black line) in the medium soil.

borne response spectra $v=150 \mathrm{~km} / \mathrm{h}$ presents higher value. The differences between both models were not strongly influenced by train speed.

\subsection{Distance from the track}

Building response due to the passage of a S-100 train travelling at $v=150 \mathrm{~km} / \mathrm{h}$ was analysed for different distances between the track to the building. The moderately stiff soil type was again considered. Figure 15 shows the vertical ground-borne response spectra $\Lambda_{i}^{j}$ computed from the free-field vibration. As expected, ground-borne vibration levels were increasingly damped with increasing distance from the track.

Figure 16 shows the effect of the distance from the track to the building on the overall RMS value of the response, where it is seen that the building response decreases with increasing distance. This correlation 


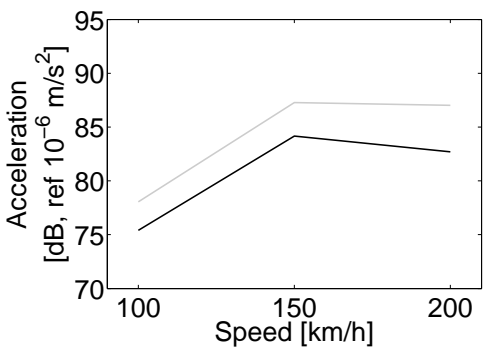

(a)

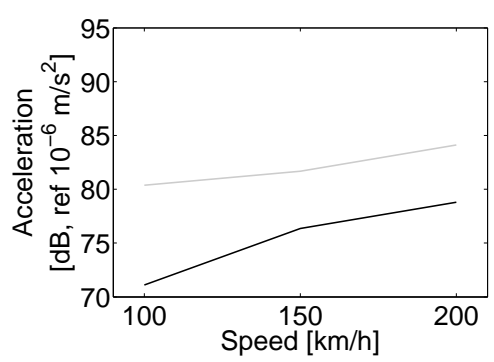

(d)

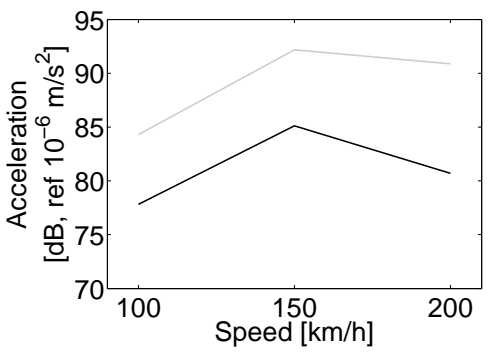

(b)

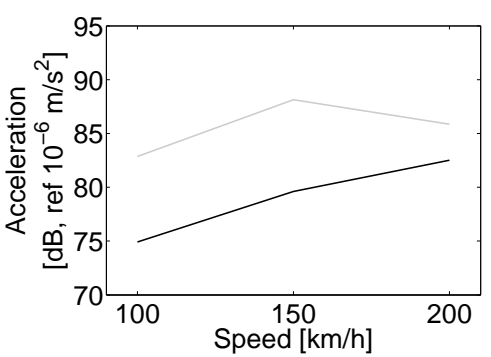

(e)

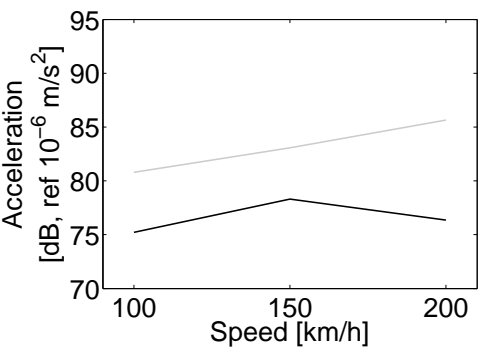

(c)

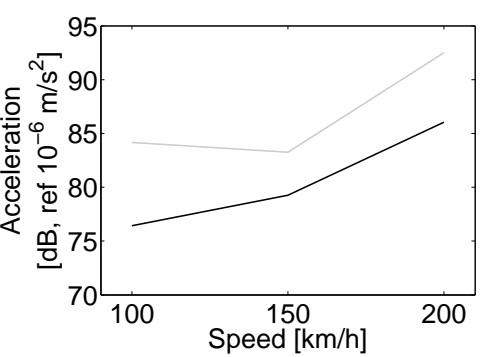

(f)

Figure 14: Overall RMS value of the acceleration response due to a train passage at different speeds evaluated at the observation points (a,b,c) $A$ and (d,e,f) $B$ computed from the SSIFiBo toolbox (black line) and the scoping model (Equation (24)) (grey line) for the (a,d) four-storey building, (b,e) eight-storey building and $(\mathrm{c}, \mathrm{f})$ twelve-storey building.

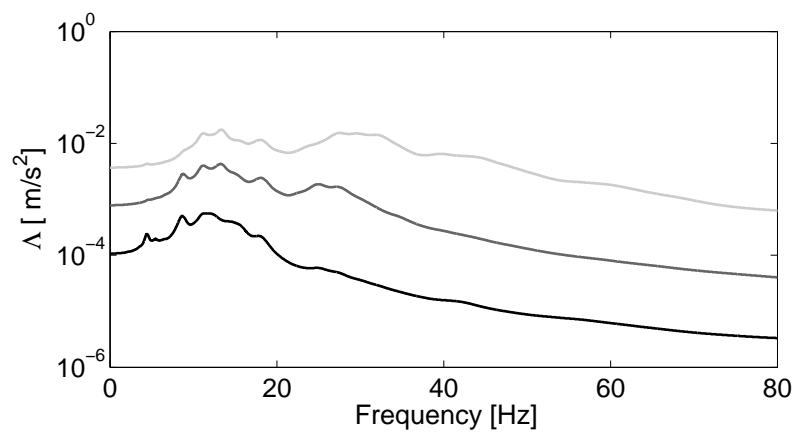

Figure 15: Vertical ground-borne response spectra $\Lambda_{i}^{j}$ at $20 \mathrm{~m}$ (light grey line), $40 \mathrm{~m}$ (dark grey line) and $70 \mathrm{~m}$ (black line) from the track center due to a train passage at $v=150 \mathrm{~km} / \mathrm{h}$.

between distance from the track and the response both in the free field and in the building is consistent with previous research [41]. The scoping model predicted elevated values with regard to the SSIFiBo model, however, the accuracy of the scoping model remained broadly constant with distance. 


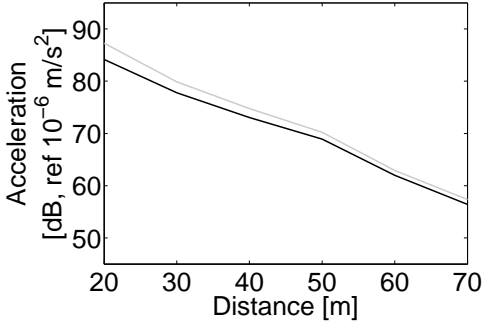

(a)

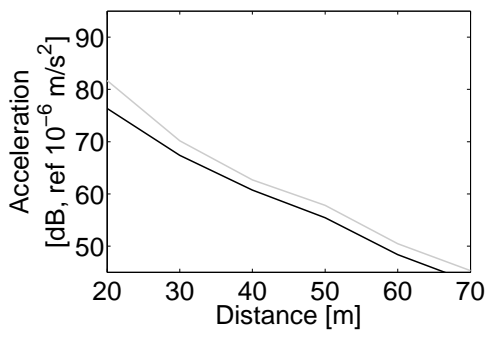

(d)

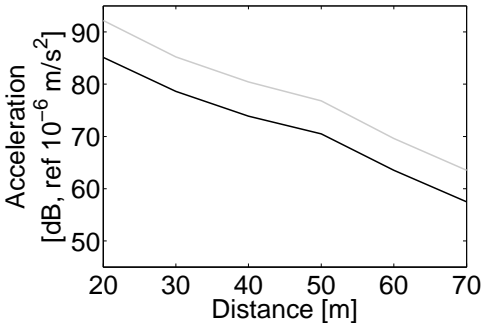

(b)

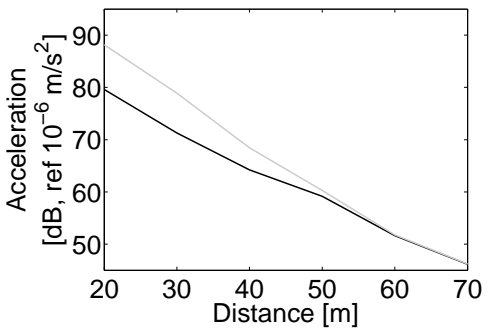

(e)

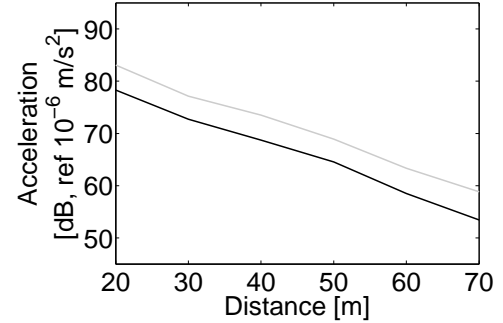

(c)

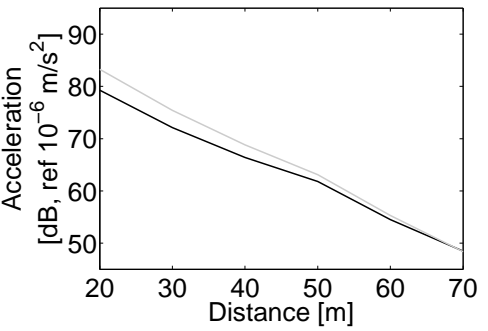

(f)

Figure 16: Overall RMS value of the acceleration response evaluated at the observation points (a,b,c) $A$ and (d,e,f) $B$ computed from the SSIFiBo toolbox (black line) and the scoping model (Equation (24)) (grey line) for the $(\mathrm{a}, \mathrm{d})$ four-storey building, $(\mathrm{b}, \mathrm{e})$ eight-storey building and $(\mathrm{c}, \mathrm{f})$ twelve-storey building.

\subsection{Remarks of the sensitivity analysis}

The overall RMS value of the acceleration for the 162 problems were computed using both models (scoping and SSIFiBo) to assess the accuracy of the proposed methodology. The difference between the responses computed from both models was calculated as:

$$
\Delta a_{R M S}[\mathrm{~dB}]=20 \log \left(\frac{a_{R M S}^{P}}{a_{R M S}^{S}}\right)
$$

where $a_{R M S}^{P}$ and $a_{R M S}^{S}$ were the responses computed by the proposed model and the SSIFiBo toolbox, respectively. Figure 17 shows this difference for the 162 problems evaluated at the observation points $A$ and $B$ at all the storey levels that correspond with 2592 cases. It can be seen that the difference between both models is normally distributed (Figure 17.(a)) with mean value $\mu=3 \mathrm{~dB}$ and standard deviation $\sigma=2.6 \mathrm{~dB}$ (Figure 17.(b)).

Figure 18 presented all the cases evaluated. The confidence region $\left[a_{R M S}^{S}+\mu \pm 2 \sigma\right]$ and the expected value $a_{R M S}^{S}+\mu$ are superimposed. It was found that $96.45 \%$ of the results were within this confidence region, and that most of the results from the scoping model were higher in magnitude than those obtained from the detailed model. The uncertainty of the predictions from the scoping model were within a range between $-3 \mathrm{~dB}$ to $11 \mathrm{~dB}$ and thus similar to the $5 \mathrm{~dB}-20 \mathrm{~dB}$ values found in previous research [43-45]. 

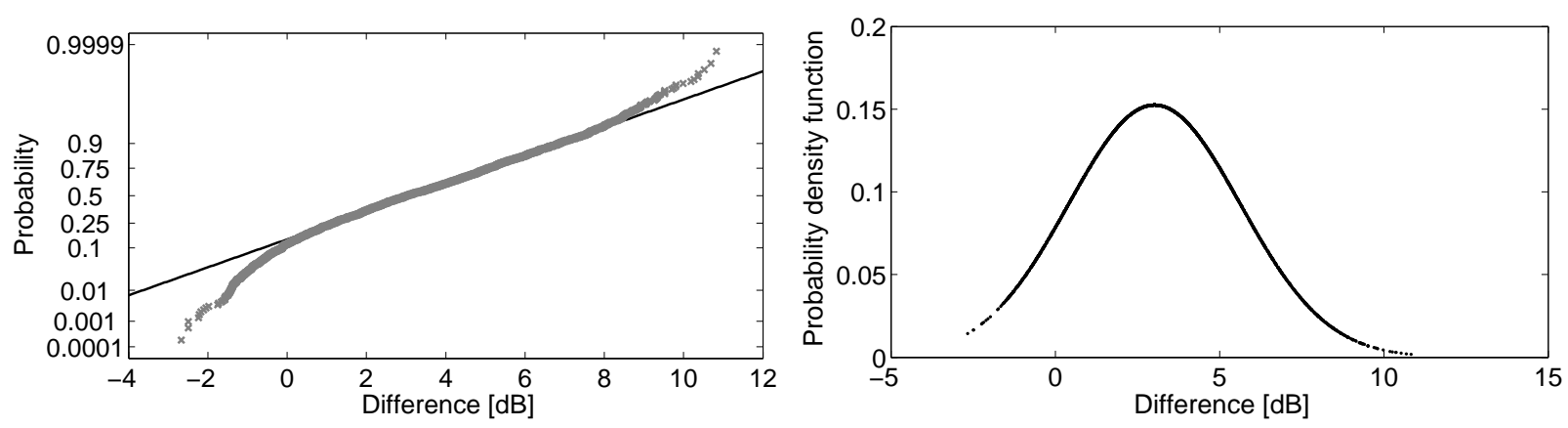

Figure 17: (a) Distribution of the difference between both models (grey crosses) against the normal distribution (black line) and (b) probability density function of the difference.

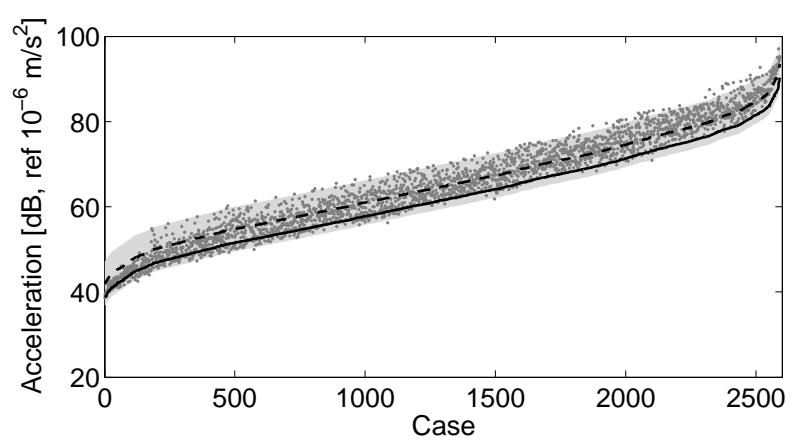

Figure 18: Overall RMS of the building for the 162 problems evaluated at the observation points $A$ and $B$ computed by the scoping model (grey points) and from the SSIFiBo toolbox (black line). Superimposed are the confidence region (grey area) and the expected value (black dashed line).

The sensitivity analysis showed that soil material properties were a relevant parameter that could affect the accuracy of the vibration level prediction, due to the deviation shown in Figure 10.

One of the advantages of the proposed method is its computational efficiency. Table 5 shows the computational cost to obtain the results of the twelve-storey response for a S-100 train travelling at $v=150 \mathrm{~km} / \mathrm{h}$ using an Intel Core $1701.87 \mathrm{GHz}$ computer. The running time shown refers to the immission problem of waves in the building. The cost needed to compute the BEM model in the SSIFiBo toolbox, the groundborne response $\Lambda_{i}^{j}$ in the scoping model and the FEM model of the building were not included. The difference between the running time required in both models was due to the more comprehensive BEM-FEM methodology used by the SSIFiBo toolbox to consider the SSI against the simple FEM procedure of the scoping model. The time using the proposed scoping model is much lower than the necessary for the detailed prediction model (between 45-135 times faster depending on soil stiffness). Therefore, the scoping model could be a powerful tool during the early design stages of railway lines where a large number of building vibrations assessment. 
Table 5: Average running time for a S-100 travelling at $v=150 \mathrm{~km} / \mathrm{h}$ considering the twelve-storey building

\begin{tabular}{c|ccc}
\hline & \multicolumn{3}{|c}{ Average running time } \\
& Soft soil & Medium soil & Stiff soil \\
\hline SSIFiBo toolbox & $t=3 \mathrm{~h}$ & $t=7.5 \mathrm{~h}$ & $t=9 \mathrm{~h}$ \\
\hline Proposed scoping Model & \multicolumn{3}{|}{$t=4 \mathrm{~min}$} \\
\hline
\end{tabular}

\section{Conclusions}

In this paper, a scoping model to predict vibrations in buildings induced by railway traffic considering soil-structure interaction was proposed. The scoping model is attractive because the structural vibration induced by train passage can be assessed in minimal computational time.

The scoping model uses the ground-borne response spectra $\Lambda_{i}^{j}$ computed from either numerical or experimentally free field vibrations (ground motion at the three orthogonal directions should be measured). Therefore, it is useful for cases of new lines, and also existing lines where new buildings are planned. To minimise calculation times, building response is obtained using modal superposition.

The proposed model was verified against a detailed prediction model based on a BEM-FEM formulation. The agreement was good and any discrepancies were mainly due to the simplifications assumed in the proposed formulation and the different procedure to consider the structural damping in both models. Therefore it can be considered a highly effective tool for early stage prediction.

The proposed methodology was used to analyse the dynamic behaviour of a building due to train passages, considering numerically generated free-field vibrations as input data. The effect of different parameters was analysed: soil properties, building height, train speed and distance from the track to the building. The building response showed a clear dependence on these parameters.

In conclusion, the scoping model allows engineers and designers to evaluate building response due to train passage at the early design stage with confidence. The proposed model involves a powerful tool easily implementable in general purpose commercial FEM software. The contribution of the dominant frequencies obtained using the scoping model were in good agreement with those obtained using a detailed design model, and the estimation of the overall RMS acceleration values were also strong. Generally, the new model provides conservative predictions of overall RMS values of the acceleration, with typical discrepancies between $-3 \mathrm{~dB}+11 \mathrm{~dB}$.

\section{Acknowledgments}

This research was funded by the Spanish Ministry of Economy and Competitiveness (Ministerio de Economía y Competitividad) through research project BIA2013-43085-P. Financial support is gratefully acknowledged. The support given by the Andalusian Scientific Computing Centre (CICA) is also gratefully. 


\section{References}

[1] D.P. Connolly, G.P. Marecki, G. Kouroussis, I. Thalassinakis, P.K. Woodward, The growth of railway ground vibration problems - A review, Science of the Total Environment (2015) http://dx.doi.org/10.1016/j.scitotenv.2015.09.101.

[2] International Organization for Standardization, ISO 2631-1:2003: Mechanical vibration and shock-Evaluation of human exposure to whole-body vibration-Part 1: General requirements (2003).

[3] International Organization for Standardization, ISO 2631-2:2003: Mechanical vibration and shock-Evaluation of human exposure to whole-body vibration-Part 2: Vibration in buildings (1-80 Hz) (2003).

[4] International Organization for Standardization, ISO 14837-1:2005 Mechanical vibration-Ground-borne noise and vibration arising from rail systems-Part 1: General guidance (2005).

[5] G. Lombaert, G. Degrande, J. Kogut, S. François, The experimental validation of a numerical model for the prediction of railway induced vibrations, Journal of Sound and Vibration 297 (2006) 512-535.

[6] G. Lombaert, G. Degrande, Ground-borne vibration due to static and dynamic axle loads of InterCity and high-speed trains, Journal of Sound and Vibration 319 (2009) 1036-1066.

[7] L. Auersch, The excitation of ground vibration by rail traffic: theory of vehicle-track-soil interaction and measurements on high-speed lines, Journal of Sound and Vibration 284 (2005) 103-132.

[8] X. Sheng, C.J.C. Jones, D.J. Thompson, Prediction of ground vibration from trains using the wavenumber finite and boundary element methods, Journal of Sound and Vibration 293 (2006) 575-586.

[9] P. Galvín, S. François, M. Schevenels, E. Bongini, G. Degrande, G. Lombaert, A 2.5D coupled FE-BE model for the prediction of railway induced vibrations, Soil Dynamics and Earthquake Engineering 30 (2010) 1500-1512.

[10] S. François, M. Schevenels, P. Galvín, G. Lombaert, G. Degrande, A 2.5D coupled FE-BE methodology for the dynamic interaction between longitudinally invariant structures and a layered halfspace, Computer Methods in Applied Mechanics and Engineering 199 (2010) 1536-1548.

[11] P. Alves Costa, R. Calada, A. Silva Cardoso, Track-ground vibrations induced by railway traffic: In-situ measurements and validation of a 2.5D FEM-BEM model, Soil Dynamics and Earthquake Engineering 32 (2012) 111-128.

[12] A. Romero, A. Tadeu, P. Galvín, J. António, 2.5D coupled BEM-FEM used to model fluid and solid scattering wave, International Journal for Numerical Methods in Engineering 101 (2015) 148-164.

[13] P. Jean, C. Guigou, M. Villot, A 2.5D BEM Model for Ground-Structure Interaction, Building Acoustics 11 (3) (2004) $1-17$.

[14] L. Auersch, A. Romero, P. Galvín, Respuesta dinámica de edificaciones producida por campos de onda incidentes considerando la interacción suelo-estructura, Revista Internacional de Métodos Numéricos para Cálculo y Diseño en Ingeniería 30 (4) (2014) 256-263.

[15] P. Galvín, J. Domínguez, High-speed train-induced ground motion and interaction with structures, Journal of Sound and Vibration 307 (2007) 755-777.

[16] H. Xia, Y.M. Cao, G. De Roeck, Theoretical modeling and characteristic analysis of moving-train induced ground vibrations, Journal of Sound and Vibration 329 (2010) 819-832.

[17] P. Galvín, A. Romero, J. Domínguez, Fully three-dimensional analysis of high-speed train-track-soil-structure dynamic interaction, Journal of Sound and Vibration 329 (2010) 5147-5163.

[18] P. Lopes, P. Alves Costa, M. Ferraz, R. Calada, A. Silva Cardoso, Numerical modeling of vibrations induced by railway traffic in tunnels: From the source to the nearby buildings, Soil Dynamics and Earthquake Engineering 61-62 (2014) $269-285$

[19] P. Coullier, G. Lombaert, G. Degrande, The influence of source-receiver interaction on the numerical prediction of railway induced vibrations, Journal of Sound and Vibration 333 (2014) 2520-2538. 
[20] J.T. Nelson, H.J. Sauernman, A prediction procedure for rail transportation groundborne noise and vibration, Transportation Research Record: Journal of the Transportation Research Board 1143 (1987) 26-35.

[21] C. Madshus, B. Bessason, L. Harvik, Prediction model for low frequency vibration from high speed railways on soft ground, Journal of Sound and Vibration 193 (1) (1996) 195-203.

[22] F. Rossi, A. Nicolini, A simple model to predict train-induced vibration: theoretical formulation and experimental validation, Enviornmental Impact Assesment Review 23 (2003) 305-322.

[23] C. With, M. Bahrekazemi, A. Bodare, Validation of an empirical model for prediction of train-induced ground vibrations, Soil Dynamics and Earthquake Engineering 26 (2006) 983-990.

[24] C.E. Hanson, D.A. Towers, L.D. Meister, High-speed ground Transportation Noise and Vibration Impact Assessment, HMMH Report 293630-4, U.S. Department of Transportation, Federal Railroad Administration, Office of Railroad Development.

[25] C.E. Hanson, D.A. Towers, L.D. Meister, Transit Noise and Vibration Impact Assessment, Report FTA-VA-90-1003-06, U.S. Department of Transportation, Federal Transit Administration, Office of Planning and Environment.

[26] H. Verbraken, G. Lombaert, G. Degrande, Verification of an empirical prediction method for railway induced vibrations by means of numerical simulations, Journal of Sound and Vibration 330 (8) (2011) 1692-1703.

[27] K. Kuo, H. Verbraken, G. Degrande, G. Lombaert, Hybrid predictions of railway induced ground vibration using a combination of experimental measurements and numerical modelling, Journal of Sound and Vibration 373 (2016) 263284.

[28] L. Auersch, Building Response due to Ground Vibration-Simple Prediction Model Based on Experience with Detailed Models and Measurements, International Journal of Acoustics and Vibration 15 (3) (2010) 101-112.

[29] S. François, L. Pyl, H.R. Masoumi, G. Degrande, The influence of dynamic soil-structure interaction on traffic induced vibrations in buildings, Soil Dynamics and Earthquake Engineering 27 (2007) 655-674.

[30] D.P. Connolly, G. Kouroussis, A. Giannopoulos, O. Verlinden, P.K. Woodward, M.C. Forde, Assesment of railway vibrations using an efficient scoping model, Soil Dynamics and Earthquake Engineering 58 (2014) 37-47.

[31] D.P. Connolly, G. Kouroussis, P.K. Woodward, A. Giannopoulos, O. Verlinden, M.C. Forde, Scoping prediction of reradiated ground-borne noise and vibration near high speed rails lines with variable soils, Soil Dynamics and Earthquake Engineering 66 (2014) 78-88.

[32] R. Clough, J. Penzien, Dynamic of Structures, McGraw-Hill, New York, 1975.

[33] X. Zhou, K. K. Tamma, Design, analysis, and synthesis of generalized single step single solve and optimal algorithms for structural dynamics, International Journal for Numerical Methods in Engineering 59 (2004) 597-668.

[34] Federal Railroad Administration, High-Speed Ground Transportation Noise and Vibration Impact Assessment, U.S. Department of Transportation (2012).

[35] Deutsches Institut für Normung, DIN 45672 Teil 2: Schwingungsmessungen in der Umgebung von Schienenverkehrswegen: Auswerteverfahren (1995).

[36] B. Davies, Integral Transforms and Their Applications, Springer Science, New York, 1985.

[37] L. Auersch, Dynamic stiffness of foundations on inhomogeneous soils for a realistic prediction of vertical building resonance, Journal of Geotechnical and Geoenvironmental Engineering 134 (3) (2008) 328-340.

[38] L. Auersch, Wave propagation in the elastic half-space due to an interior load and its application to ground vibration problems and buildings on pile foundations, Soil Dynamics and Earthquake Engineering 30 (2010) 925-936.

[39] P. Fiala, G. Degrande, F. Augusztinovicz, Numerical modelling of ground-borne noise and vibration in buildings due to surface rail traffic, Journal of Sound and Vibration 301 (2007) 718-738.

[40] P. Galvín, A. Romero, A MATLAB toolbox for soil-structure interaction analysis with finite and boundary elements, Soil Dynamics and Earthquake Engineering 57 (2014) 10-14. 
[41] M. Villot, P. Ropars, P. Jean, E. Bongini, F. Poisson, Modeling the influence of structural modifications on the response of a building to railway vibration, Noise Control Engineering Journal 11 (2011) 641-651.

[42] P. Alves Costa, A. Colao, R. Calada, A. Silva Cardoso, Critical speed of railway tracks. Detailed and simplified approaches, Transportation Geotechnics 2 (2015) 30-46.

[43] G. Lombaert, P. Galvín, S. François, G. Degrande, Quantification of uncertainty in the prediction of railway induced ground vibration due to the use of statistical track unevenness data, Journal of Sound and Vibration 333 (2014) 4232-4253.

[44] S. Jones, K. Kuo, M.F.M. Hussein, H.E.M Hunt, Prediction uncertainties and inaccuracies resulting from common assumptions in modelling vibration from underground railways, Proceedings of the Institution of Mechanical Engineers, Part F: Journal of Rail and Rapid Transit 226 (2012) 501-512.

[45] D.P. Connolly, P. Alves Costa, G. Kouroussis, P. Galvín, P.K. Woodward, O. Laghrouche, Large scale international testing of railway ground vibrations across Europe, Soil Dynamics and Earthquake Engineering 71 (2015) 1-12. 